\title{
Multi-objective ligand-protein docking with particle swarm optimizers
}

\author{
José García-Nieto, Esteban López-Camacho, María Jesús García-Godoy, \\ Antonio J. Nebro, José F. Aldana-Montes
}

Dept. de Lenguajes y Ciencias de la Computación, University of Malaga, ETSI Informática, Campus de Teatinos, Malaga 29071, Spain

Keywords:

Multi-objective optimization Particle swarm optimization Molecular docking

Archiving strategies

Algorithm comparison

\begin{abstract}
$\begin{array}{llllllllllllll}\text { A } & \text { B } & S & T & R & A & C & T\end{array}$
In the last years, particle swarm optimizers have emerged as prominent search methods to solve the molecular docking problem. A new approach to address this problem consists in a multi-objective formulation, minimizing the intermolecular energy and the Root Mean Square Deviation (RMSD) between the atom coordinates of the cocrystallized and the predicted ligand conformations. In this paper, we analyze the performance of a set of multiobjective particle swarm optimization variants based on different archiving and leader selection strategies, in the scope of molecular docking. The conducted experiments involve a large set of 75 molecular instances from the Protein Data Bank database (PDB) characterized by different sizes of HIV-protease inhibitors. The main moti-vation is to provide molecular biologists with unbiased conclusions concerning which algorithmic variant should be used in drug discovery. Our study confirms that the multi-objective particle swarm algorithms SMPSOhv and MPSO/D show the best overall performance. An analysis of the resulting molecular ligand conformations, in terms of binding site and molecular interactions, is also performed to validate the solutions found, from a biological point of view.
\end{abstract}

\section{Introduction}

In the last decade, a series of studies have appeared in which metaheuristics [10,12,13,23,32,34], and in particular swarm intelligence algorithms [11,16,24], have been applied to the molecular docking problem. This is a complex optimization problem based on the prediction of the position and the orientation of a small molecule (ligand) to a receptor (macromolecule) with the minimum binding energy. Molecular docking is modeled by adjusting the position variables corresponding to coordinates of translation and torsion movements of the ligand in a macromolecule's binding site.

In a previous work [24], a new multi-objective approach has been proposed in which two different objectives are to be minimized: (1) the intermolecular energy that corresponds to the ligand-receptor affinity, and (2) the Root-Mean-Square-Deviation (RMSD) score, which measures the average distance between the atom coordinates of cocrystallized and predicted ligands. The latter objective leads the algorithms to guide the search when the co-crystallized ligand is known, which complements the binding energy function. In the study, a set of prominent multi-objective evolutionary algorithms (MOEAs), i.e.,
NSGA-II, SMPSO, GDE3, MOEA/D, as well as the AutoDock base optimizer Lamarckian Genetic Algorithm (LGA), were compared, resulting the Speed Modulation Particle Swarm Optimization (SMPSO) [28] the most salient optimizer for the molecular docking instances tested. SMPSO is a multi-objective particle swarm optimization (MOPSO) approach characterized by a limitation mechanism of particles' velocities to avoid the movement of particles beyond the problem ranges. It uses an external archive to store non-dominated solutions according to the crowding distance [7], which is also referenced in the leader selection mechanism.

In the present study, our motivation is to go one step beyond the application of SMPSO for molecular docking, so we propose and evaluate new variants of this algorithm that use different archiving strategies (hypervolume, cosine distance, and aggregation) and consequently different strategies for the selection of leaders, in the scope of an extensive set of molecular instances. We here extend our previous research [11], by assessing the performance of the proposed versions of SMPSO when tackling a large benchmark of 75 PDB complexes, involving HIVprotease macromolecules and ligands from the benchmark provided by 
Ref. [26] to validate the AutoDock 4.2 energy scoring function. ${ }^{2}$ In addition, MPSO/D [3] and OMOPSO [6] algorithms are also used in the comparisons. The former is used as it is a recent MOPSO approach with prominent behavior on complex multi-objective benchmarking testbeds [3]; the later is an early reference multi-objective particle swarm optimizer in the state of the art [6]. The algorithms evaluated constitute a varied set of multi-objective particle swarm optimizers, performing different learning procedures and inducing different behaviors.

Our main goal therefore is to provide new algorithmic variants of SMPSO well-adapted to molecular docking, as well as to obtain unbiased conclusions concerning which of them (and other related MOPSOs) should be used by experts in studies in silico to find new candidate drugs for therapeutic targets. As an additional contribution, an analysis on the molecular inhibitors-receptors interactions from the resulting predicted conformations is provided to show, in terms of real use cases, how the solutions obtained are validated from a biological point of view.

The remainder of this article is as follows. Section 2 reviews the current state-of-the-art. Section 3 describes the molecular docking problem from a multi-objective formulation. The algorithms under study are described in Section 4. Section 5 reports the experimentation methodology and Section 6 analyzes the results. Finally, Section 7 discusses concluding remarks and future lines of research.

\section{Literature review}

The application of metaheuristics as search methods to optimize the binding energy in molecular docking has been studied in the past. A reference approach is the Lamarckian Genetic Algorithm (LGA) included in AutoDock, which has been widely used by the scientific community [25]. This approach focuses on optimizing the final binding energy as a single-objective function. Atilgan et al. [2] proposed AutoDockX that incorporates a sustainable GA, namely Age-Layered Population Structure (ALPS), including the age attribute for individuals. Several adaptations of the original Particle Swarm Optimization (PSO) [18] have been proposed to tackle with molecular docking. SODOCK [4] is a hybrid PSO with Solis and Wets local search procedure to optimize the energy function of AutoDock 3.05. Two other PSO approaches are: the varCPSO-ls, an extension of the CPSO algorithm with a local optimizer which is embedded inside the AutoDock 3 source code and uses its energy function [27]; and the FIPSDock algorithm, which adopts the AutoDock 4.2 energy function [19].

In the last years, several studies have appeared based on multiobjective approaches. In 2006, Oduguwa et al. [32] carried out a study based on the evaluation of three evolutionary multi-objective optimization algorithms (NSGA-II, PAES and SPEA) to minimize three objectives: 1) the internal energy of the ligand (the intramolecular energy), 2) the energy of the ligand-macromolecule interaction (the intermolecular energy) and 3) the shape of the macromolecule. These algorithms were evaluated on three PDB (Protein Data Bank) complexes. Grosdidier et al. [12] proposed two energy scoring functions and an optimization technique based on the minimization of solvation free energy of the complex, giving rise to an efficient method able to converge with a very limited number of docking evaluations. Jason et al. [16] implemented the ClustMPSO to tackle the molecular docking as a multidimensional problem by minimizing the intermolecular (the difference of the bound and unbound states of the ligand-macromolecule complex) and intramolecular (the difference of the bound and unbound states of the ligand) energies. Sandoval-Pérez et al. [34] used a multi-objective algorithm implemented in the jMetal framework to optimize the energy contributions of the unbound and bound energies of four PDB complexes. These studies have a common thread in that they do not use

\footnotetext{
${ }^{2}$ The generated benchmark of 75 PDB structures is online available and adapted to be reproduced using compatible software http://khaos.uma.es/ AutodockjMetal/instances.jsp.
}

flexible receptors (receptors without applied flexibility in the residues of their active sites) and their experiments conducted on limited sets of instances.

More recently, in Ref. [10] a set of representative multi-objective algorithms were evaluated: two variants of NSGA-II, the third evolution step of generalized differential evolution (GDE3), the multi-objective evolutionary algorithm based on decomposition (MOEA/D) and the Smetric evolutionary multi-objective optimization (SMS-EMOA). Following the optimization model proposed in Ref. [16], the objectives consisted in minimizing the intermolecular and intramolecular energies. This study involved 11 instances of ligands of different sizes and flexible side-chains of the ARG8 in the active sites of the HIV-proteases. In addition, two use cases with the epidermal growth factor receptor and the anti-angiogenic compound aeroplysinin-1 were analyzed to show the applicability of this approach in drug discovery. In 2016, LópezCamacho et al. [24] proposed a new multi-objective approach to minimize: the intermolecular energy and the RMSD (Root Mean Square Deviation) as the two objectives to evaluate the quality of the ligandprotein interactions. The molecular instances used were the same as those in Ref. [26]. The results demonstrated that SMPSO showed the best overall behavior in terms of binding energy and RMSD scores. These results are useful in those cases in which the crystallographic structure has been obtained and therefore, the ligand conformation to the receptor is known.

Finally, a recent approach is proposed in 2018 by Leonhart et al. [20], which consists in a Biased Random Key Genetic Algorithm as a sampling strategy to explore the protein-ligand conformational space. The approach is called DockThor and bases on our jMetal approach, but using in this case a single-objective formulation by minimizing the final binding energy of the ligand-macromolecule complex $(\Delta \mathrm{G})$ and adopting the energy scoring function of AutoDock Vina [21] as evaluation function.

As stated, our aim here is to follow our current line of research on the evaluation of new SMPSO proposals, although in the scope of a large and well-grounded set of molecular compounds that can help us discard biased observations.

\section{Molecular docking}

Molecular docking processes aim to finding an optimized conformation between the ligand $(L)$ and the receptor $(R)$ that results in a minimum binding energy. The interaction between $L$ and $R$ can be described by an energy function calculated from three components representing degrees of freedom: (1) the translation of the ligand molecule, involving the three axis values $(x, y, z)$ in Cartesian coordinate space; (2) the ligand orientation, modeled as a four variables quaternion including the angle slope $(\theta)$; and (3) the flexibilities, represented by the free rotation of torsion (dihedral angles) of the ligand and side-chains of the receptor's aminoacids.

- Solution Vector Representation: Each problem solution is then encoded by a real-value vector of $7+n$ variables, in which the first three values correspond to the ligand translation, the next four values correspond to the ligand and/or receptor orientation, and the remaining $n$ values are the ligand and macromolecule torsion dihedral angles (in this study $n=23$ ). Fig. 1 illustrates the solution encoding followed in this approach. To reduce the computational cost, a grid-based methodology has been applied where the proteinligand interaction site is embedded in a 3D rectangular grid, and taking into account all the protein atoms [14]. In this way, the protein contribution at any given point is obtained by tri-linear interpolation in the grid cell. As a consequence of this process, the range of translation variables $(x, y, z)$ is $[0 \cdots 120]$, so it has been delimited between the limits of the coordinates of the grid space previously set for each problem. Orientation (quaternion) and torsion variables are measured in radians and encoded in the range of $[-\pi, \pi]$. 

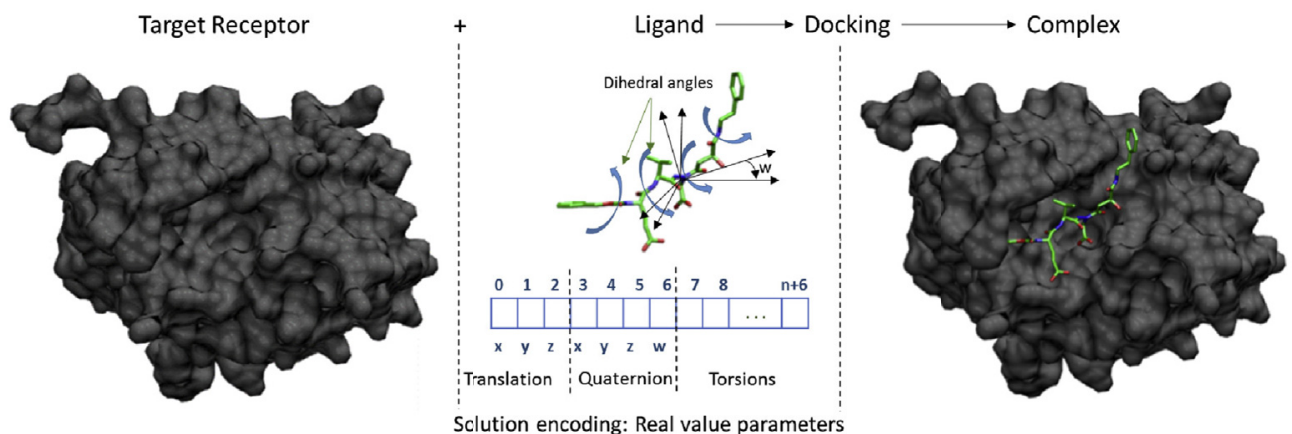

Fig. 1. Solution encoding. The first three values (translation) are the coordinates of the center of rotation of the ligand. The next four values correspond to quaternion and $(\theta)$. The rest of the values represent the freedom degrees of the ligand and macromolecule when flexibility is applied to these two molecules.

- Multi-objective evaluation: the bi-objective formulation used here consists of: the $E_{\text {inter }}$ and the RMSD score. The $E_{\text {inter }}$ is the energy function as follows:

$E_{\text {inter }}=Q_{\text {bound }}^{R-L}+Q_{\text {unbound }}^{R-L}$

$$
\begin{aligned}
Q= & W_{v d w} \sum_{i, j}\left(\frac{A_{i j}}{r_{i j}^{12}}-\frac{B_{i j}}{r_{i j}^{6}}\right)+W_{h b o n d} \sum_{i, j} E(t)\left(\frac{C_{i j}}{r_{i j}^{12}}-\frac{D_{i j}}{r_{i j}^{10}}\right)+ \\
& +W_{\text {elec }} \sum_{i, j} \frac{q_{i} q_{j}}{\varepsilon\left(r_{i j}\right) r_{i j}}+W_{\text {sol }} \sum_{i, j}\left(S_{i} V_{j}+S_{j} V_{i}\right) e^{\left(-r_{i j}^{2} / 2 \sigma^{2}\right)}
\end{aligned}
$$

$Q_{\text {bound }}^{R-L}$ and $Q_{\text {unbound }}^{R-L}$ are the states of bound and unbound of the ligand-receptor complex, respectively. Each pair of energetic evaluation terms includes evaluations $(Q)$ of dispersion and repulsion $(v d w)$, hydrogen bonds (hbond), electrostatics (elec) and desolvation (sol). Weights $W_{v d w}, W_{\text {hbond }}, W_{\text {conf }}, W_{\text {elec }}$, and $W_{\text {sol }}$ of Equation (2) are constants for Van der Waals, hydrogen bonds, torsional forces, electrostatic interactions and desolvation, respectively. The interatomic distance is represented by $r_{i j}, A_{i j}$ and $B_{i j}$ in the first term are Lennard-Jones parameters taken from the Amber force field. Similarly, $C_{i j}$ and $D_{i j}$ in the second term are Lennard-Jones parameters for maximum well depth of potential energies between two atoms, and $E(t)$ represents the angledependent directionality. The third term uses a Coulomb approach for electrostatics. Finally, the fourth term is calculated from the volume $(V)$ of the atoms that surround a given atom weighted by $S$, and an exponential term which involves atom distances. An extended explanation of all these variables can be found in Ref. [26].

The second objective, RMSD, measures the average distance between the known ligand position (obtained from in vivo experiments) in the receptor and the computed position of the docking ligand, that takes into account symmetry, partial symmetry (e.g. symmetry within a rotatable branch) and near-symmetry in a simple heuristic way. Ideally, the lower the RMSD score the better the solution. As mentioned, a ligand-receptor docking solution with an RMSD score below $2 \AA$ is considered as a solution with high docking accuracy [21]. The RMSD for two structures $a$ and $b$ is:

$$
\begin{aligned}
R M S D_{a b} & =\max \left(R M S D_{a b}^{\prime}, R M S D_{b a}^{\prime}\right), \\
\text { with } R M S D_{a b}^{\prime} & =\sqrt{\frac{1}{N} \sum_{i} \min _{\mathbf{j}} r_{2}^{i j}}
\end{aligned}
$$

The sum is over all $N$ heavy atoms in structure $a$, the minimum is over all atoms in structure $a$ with the same element type as atom $i$ in structure $b$.

\section{Algorithms}

The algorithmic variants studied can be classified as Pareto-based Multi-Objective Particle Swarm Optimizers (MOPSOs). The basic idea, commonly found in all these algorithms, is to select, as leaders, those particles that are non-dominated with respect to the swarm. However, this leader selection scheme can differ slightly depending on which archiving strategy each algorithm implements (e.g., information provided by a density estimator). We now summarize the main features of the MOPSOs under consideration:

\subsection{SMPSO}

It is an algorithm following the classic particle swarm algorithm metaheuristic [18], so it manages a set of solutions or particles which are referred to as the swarm. The position of particle $\mathbf{x}_{i}$ at the generation $t$ is updated with Equation (4):

$\mathbf{x}_{i}(t)=\mathbf{x}_{i}(t-1)+\mathbf{v}_{i}(t)$

where the factor $\mathbf{v}_{i}(t)$ is known as velocity, and it is defined as:

$\mathbf{v}_{i}(t)=\omega \cdot \mathbf{v}_{i}(t-1)+C_{1} \cdot r_{1} \cdot\left(\mathbf{x}_{p_{i}}-\mathbf{x}_{i}\right)+C_{2} \cdot r_{2} \cdot\left(\mathbf{x}_{g_{i}}-\mathbf{x}_{i}\right)$

In Equation (4), $\mathbf{x}_{p_{i}}$ is the best solution that $\mathbf{x}_{i}$ has viewed, $\mathbf{x}_{g_{i}}$ is the best particle (known as the leader) that the entire swarm has viewed, $\omega$ is the inertia weight of the particle and controls the trade-off between global and local influence, $r_{1}$ and $r_{2}$ are two uniformly distributed random numbers in the range [0,1], and $C_{1}$ and $C_{2}$ are specific parameters which control the effect of the personal and global best particles. The key feature of SMPSO is the constriction coefficient (Equation (6)) it uses that is obtained from the constriction factor $\chi$ originally developed by Clerc and Kennedy (Equation (5)) in Ref. [5]. This mechanism makes the SMPSO perform successfully on multi-modal large scale problems [8]. The constriction coefficient is defined as:

$\chi=\frac{2}{2-\varphi-\sqrt{\varphi^{2}-4 \varphi}}$

where

$\varphi= \begin{cases}C_{1}+C_{2} & \text { if } C_{1}+C_{2}>4 \\ 0 & \text { if } C_{1}+C_{2} \leq 4\end{cases}$

Additionally, this algorithm further bounds the accumulated velocity of each variable $j$ (in each particle) by means of the following velocity constriction equation:

$v_{i, j}(t)= \begin{cases}\delta_{j} & \text { if } v_{i, j}(t)>\delta_{j} \\ -\delta_{j} & \text { if } v_{i, j}(t) \leq-\delta_{j} \\ v_{i, j}(t) & \text { otherwise }\end{cases}$

where

$\delta_{j}=\frac{\left(\text { upper_limit }_{j}-\text { lower_limit }_{j}\right)}{2}$

As commented before, SMPSO adopts the use an external bounded archive to store the non-dominated solutions found during the search 
[28]. The archive contains the current Pareto front approximation found by the algorithm, and it applies the crowding distance density estimator [7] to decide which particle to remove when it is full. The archive is also used in the leader strategy selection, comprising a binary tournament based on randomly selecting two solutions from it and taking the one with the highest crowding distance value (i.e., the one located in the least crowded region of the front composed by all archived solutions). The maximum size of the archive of leaders is fixed equal to the size of the swarm. After each generation, the set of leaders is updated, and so are the corresponding crowding values. If the size of the set of leaders is greater than the maximum allowable size, only the best leaders are kept based on their crowding values. The rest of the leaders are discarded. SMPSO uses the crowding factor to select the leaders, as well as to fix the size of the external archive of leaders.

A perturbation, implemented as a mutation operator, is also incorporated. Its pseudo-code is included in Algorithm 1. The local best position of a particle $i$ is obtained by applying a dominance test with the rest of the particles in the swarm in such a way that the current best particle (which initially is particle $i$ ) is updated when it is dominated by another one.

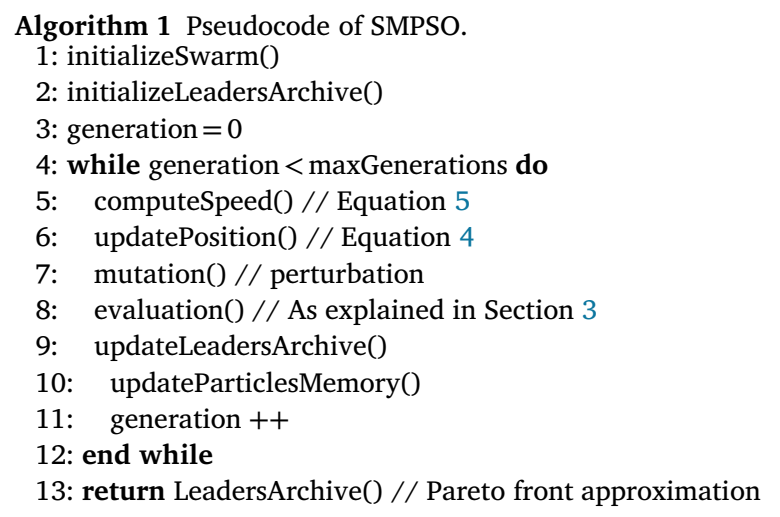

\subsection{SMPSOhv}

This variant was presented in Ref. [29], where a study of different leader selection mechanisms on SMPSO was conducted. SMPSO ${ }_{h v}$ is characterized by the replacing method based on the crowding distance. Instead, this method uses the degree of contribution of the solutions in the external archive according to the hypervolume indicator [39] $\left(I_{H V}\right)$.

This indicator calculates the $n$-dimensional space covered by members of a non-dominated set of solutions $Q$, e.g., the region enclosed by the discontinuous line in Fig. 2, $Q=\{A, B, C\}$, for problems where all objectives are to be minimized. Mathematically, for each solution $i \in Q$, a hypercube $v_{i}$ is constructed with a reference point $W$ and the solution $i$ as the diagonal corners of the hypercube. The reference point can simply be found by constructing a vector of worst objective function values. Thereafter, a union of all hypercubes is found, and its hypervolume $\left(I_{H V}\right)$ is calculated:

$I_{H V}=\operatorname{volume}\left(\bigcup_{i=1}^{|Q|} v_{i}\right)$

Solution fronts with large values of $I_{H V}$ are desirable, so the underlying idea in this variant lies on selecting as a leader one of the particles contributing the most to the hypervolume of the Pareto front approximation, computed so far by the algorithm. To apply this scheme, the archive of leaders is changed for an archive managed by the contribution of each solution to the value of this indicator. This archive works as the one described for SMPSO but, when the archive becomes full, instead of discarding the solution with the smallest crowding distance, we choose the solution contributing the least to the hypervolume. In this variant, when the velocity of a particle has to be updated, two solu-

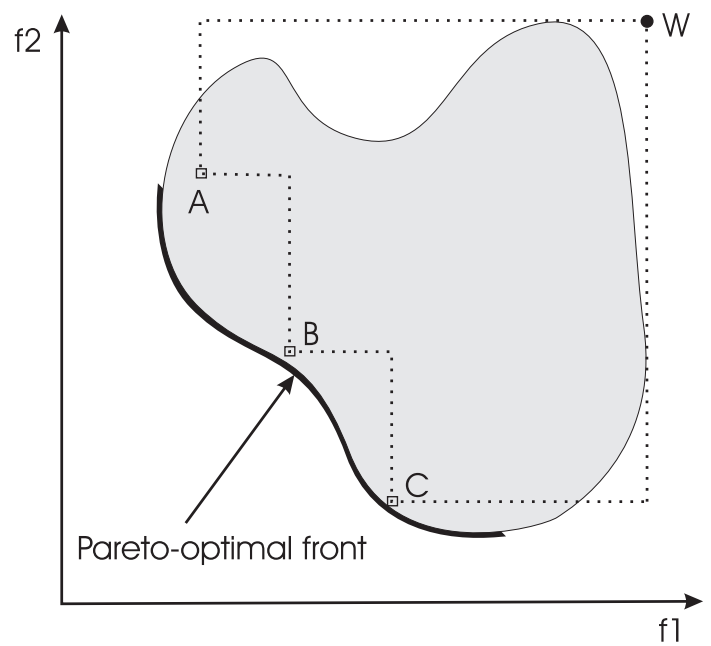

Fig. 2. The hypervolume (delimited by the dotted line) enclosed by the nondominated solutions.

tions are randomly selected from the archive, and the one contributing the most to the archive's hypervolume is selected as the leader.

\subsection{SMPSOC}

This is a recent variant presented in 2016 [11] with the key feature of using the cosine similarity as density estimator to organize nondominated solutions in the external archive. Cosine similarity is a measure of distance between two non-zero vectors that computes the cosine of the angle between them by using the Euclidean dot product formula as follows:

$\mathbf{a} \cdot \mathbf{b}=\|\mathbf{a}\|\|\mathbf{b}\| \cos \theta$

Given two vectors of attributes, $D$ and $F$, the $\operatorname{cosine}$ similarity, $\cos \theta$, is represented using a dot product and magnitude as:

$\cos \theta=\frac{\mathbf{D} \cdot \mathbf{F}}{\|\mathbf{D}\|\|\mathbf{F}\|}=\frac{\sum_{i=1}^{n} D_{i} F_{i}}{\sqrt{\sum_{i=1}^{n} D_{i}^{2}} \sqrt{\sum_{i=1}^{n} F_{i}^{2}}}$

This way, two vectors in the same direction have a cosine similarity value equals to zero, two perpendicular vectors have a cosine similarity value of 1 , and in-between values indicate intermediate similarity or dissimilarity.

As all the solutions in an external archive are non-dominated, we can define a density estimator by fixing a reference point and computing the cosine similarity among the vectors conformed by the archive solutions with regards to that reference point. The problem studied in this paper has two objectives, so it is possible to sort the solutions in the archive by the first objective and compute, for each solution, a density value by summing up the cosine similarity of each point to their previous and next points; extreme points have a similarity distance equals to 0 . Those points with the largest cosine density value are in the most densely populated region. An important issue in this technique is to select the proper reference point. Our previous study [24] indicated that the fronts have a convex shape, so we choose an approximation to the Nadir point, defined as $z^{\text {Nad }}=\left(z_{1}^{N a d}, \ldots, z_{k}^{\text {Nad }}\right)^{T}$, where $z_{i}^{N a d}=\max _{x \in E} f_{i}(x)$ for $i=1, \ldots, k$ (being $k$ is the number of objectives) by taking the highest objective values of the solutions in the archive.

\subsection{SMPSOD}

The fourth SMPSO version in our study is an archive-less approach. To leave out the archive, we take the strategy of designing an aggregative version of SMPSO inspired by the MOEA/D [38], where a multi- 
objective problem can be decomposed into a set of single-objective problems that can be optimized at the same time. This way, a set of evenly spread weight vectors $\lambda_{1}, \lambda_{2}, \ldots, \lambda_{N}$ are defined, being $N$ the size of the swarm. Then, each particle $\mathbf{x}_{i}$ is associated with the vector $\lambda_{i}$ and a neighborhood defined as a set of its several closest weight vectors in $\lambda_{1}, \lambda_{2}, \ldots, \lambda_{N}$. A scalarizing strategy is then applied that follows the Tchebycheff scheme, by considering the ideal reference point $z^{*}=\left(z_{1}^{*}, \ldots, z_{k}^{*}\right)^{T}$, where $z_{1}^{*}=\min _{x \in S} f_{i}(x)$ for $i=1, \ldots, k$ as

Minimize $f^{T c h}\left(\mathbf{x} \mid \lambda, z^{*}\right)=\max _{i=1, \ldots, N}\left\{\lambda_{i} \cdot\left|z_{i}^{*}-f_{i}(\mathbf{x})\right|\right\}$

Each element $z^{*}$ of the reference point $z^{*}$ is specified by the minimum (i.e., best assuming minimization) value of each objective $f_{i}(\mathbf{x})$ among the examined solutions during the execution of SMPSOD.

The strategy for obtaining the local best of a particle $\mathbf{x}_{p_{i}}$ is the same procedure as used by MOEA/D to update a neighborhood. The leader's update strategy consists in finding the best solution in the neighborhood by considering the scalar values of the particles taking into account their weight vectors. SMPSOD is originally proposed in this study and its performance is shown to be competitive with regards to the other compared variants, when solving molecular docking instances.

\subsection{OMOPSO}

Optimized MOPSO [33] includes the use of the crowding distance of NSGA-II to filter out leader solutions and the combination of two mutation operators to accelerate the convergence of the swarm. The original OMOPSO algorithm makes use of the concept of $\epsilon$-dominance to limit the number of solutions produced by the algorithm. We consider here a variant discarding the use of $\epsilon$-dominance, so the leaders archive is the result of the execution of the technique.

Therefore, for each particle, the leader is selected by means of a binary tournament based on the crowding value of the leaders. The maximum size of the set of leaders is fixed equal to the size of the swarm. After each generation, the set of leaders is updated, and so are the corresponding crowding values. If the size of the set of leaders is greater than the maximum allowable size, only the best leaders are retained based on their crowding value. The rest of the leaders are eliminated. OMOPSO calculates the crowding factor to select the leaders and uses this information to fix the size of the set of leaders (external archive). This feature considerably simplifies the mechanism to control the set of leaders without requiring any additional parameter or selection criterion. SMPSO was inspired by the OMOPSO algorithm, so we decided to include it in our comparisons as a reference multi-objective PSO in the state of the art.

\section{6. $M P S O / D$}

It is a recent MOPSO [3] algorithm based on objective space decomposition. Similarly to SMPSOD, MPSO/D is an archive-less approach that decomposes the objective space of the multi-objective optimization problem in to a set of sub-regions based on a set of direction vectors. The population is classified by means of a set of weight vectors $\lambda_{1}, \lambda_{2}, \ldots, \lambda_{N}$, being $N$ the size of the swarm. Then, each particle $\mathbf{x}_{i}$ is associated with the vector $\lambda_{i}$ and a neighborhood defined as a set of its several closest weight vectors in $\lambda_{1}, \lambda_{2}, \ldots, \lambda_{N}$. A scalarizing strategy is applied by taking into account the ideal reference point $z^{*}=\left(z_{1}^{*}, \ldots, z_{k}^{*}\right)^{T}$, where $z_{i}^{*}=\min _{x \in S} f_{i}(x)$ for $i=1, \ldots, k$ as follows:

Minimize $f^{T c h}\left(\mathbf{x}_{i} \mid \lambda_{i}, z^{*}\right)=\max _{i=1, \ldots, N}\left\{\frac{\lambda_{i} \cdot\left(f_{i}(\mathbf{x})-z_{i}^{*}\right)^{T}}{\lambda_{i} \cdot\left(f_{i}(\mathbf{x})-z_{i}^{*}\right)}\right\}$

Each element $z^{*}$ of the reference point $z^{*}$ is specified by the minimum value of each objective $f_{i}(\mathbf{x})$ among the examined solutions during the execution of MPSO/D. Then, it maintains the diversity of solutions by making each sub-region have a solution to the maximum extent.
In addition, MPSO/D adopts a series of extra schemes to improve the convergence. The crowding distance is used to calculate the fitness values of the reserved solutions for selection operator to improve the convergence of the obtained non-dominated solution set. A neighborhood strategy of particles is used to determine the leaders and local bests, which help the crossover operators to improve the search ability of the algorithm.

In summary, in our study we include OMOPSO, MPSO/D, and four SMPSO variants with different archiving strategies: crowding distance based (original SMPSO), hypervolume contribution based (SMPSO ${ }_{h v}$ ), cosine distance based (SMPSOC), and archive-less (SMPSOD). They constitute a set of classical and modern algorithms in the specific area of particle swarm optimization for multi-objective optimization, but involving different learning procedures.

\section{Experimentation}

All the SMPSO variants, as well as OMOPSO and MPSO/D, have been implemented in $\mathrm{C}++$ by following the architecture of jMetalCpp $[17,22,30],{ }^{3}$ an open-source framework of single/multi-objective optimization metaheuristics. The molecular binding procedure is performed by AutoDock 4.2 [31,26], a widely-used tool for virtual drug discovery involving rigid and flexible docking simulations. In this study, jMetalCpp and AutoDock are integrated in such a way that the algorithms available in jMetalCpp can communicate with AutoDock to cooperate in molecular docking optimization. ${ }^{4}$ This means that, whenever the binding quality of a new solution has to be numerically quantified, it is sent to AutoDock to be evaluated in terms of binding energy and RMSD. After this evaluation, AutoDock returns the corresponding objective values to the algorithm, which are assigned to the evaluated solution. This approach is efficient and flexible, allowing any of the algorithms included in jMetalCpp to be easily used to solve docking problems.

For the experimental phase, we have used an extensive set of 75 molecular complexes that were previously generated in Ref. [26], although they were adapted to be tackled by AutoDock + jMetal software. In these compounds, the flexibility of ligand is limited to 10 torsional degrees of freedom. As shown in Table 1, this set of complexes can be classified in terms of the type of ligand: small, medium, and large size inhibitors, as well as cyclic urea inhibitors. The PDB codes of these structures and the range of crystalographic resolution in $\AA$ are also shown in this table.

The docking studies carried out with these instances in Ref. [26] to test the energy function of AutoDock 4.2 demonstrated that the most difficult problems are those involving smaller ligands. This is due to the flexibility added to the receptor side-chains (ARG-8), which increases the space of ligand interactions. These instances have been taken from the PDB database, ${ }^{5}$ for which the reference conformations obtained by means of in vivo experiments are also provided. These reference conformations are then used to calculate the RMSD.

\subsection{Methodology}

For each combination of algorithm and molecular compound, 31 independent runs have been carried out to obtain the distributions of results. From these distributions, the median and interquartile range (IQR) have been computed as measures of central tendency and statistical dispersion, respectively. We have considered the Hypervolume $\left(I_{H V}\right)$ [39] and the Inverted Generational Distance Plus $\left(I_{I G D+}\right)$ [15] as quality indicators to assess the algorithm's performance. These two metrics take into account the convergence, as well as the diversity degrees of

\footnotetext{
3 In URL http://jmetalcpp.sourceforge.net/.

${ }^{4}$ In URL http://khaos.uma.es/autodockjmetal/.

${ }^{5}$ In URL: http://www.rcsb.org/pdb/home/home.do.
} 
Table 1

$\mathrm{X}$-ray crystal structure coordinates taken from the PDB database and used in docking experiments. They consist of 75 molecules with accession codes from the PDB database. The range of resolution ( $\AA$ ) of each subgroup is also shown in the last column.

\begin{tabular}{ll}
\hline Ligand type & PDB code \\
\hline Small size & 1a9m, 1aaq, 1b6l, 1b6m, 1bdl, 1bdq, 1bdr, 1gnm, 1gnn, 1gno, 1hbv, 1heg, 1hih, 1hpv, 1hsg, 1hte, 1kzk, 1sbg, 1tcx, 1zih, 1zir, 3aid \\
Medium size & 1b6j, 1b6k, 1b6p, 1d4k, 1d4l, 1hef, 1hps, 1hxw, 1izh, 1izi, 1jld, 1k6c, 1k6p, 1k6t, 1k6v, 1mtr, 1mui, 2bpv, 2bpx, 4hvp, 4phv, 5hvp \\
Large size & 1a94, 1hiv, 1hos, 1htg, 1hvi, 1hvj, 1hvk, 1hvl, 1hvs, 1hwr, 1ody, 1vij, 1vik, 3tlh, 7hvp, 8hvp, 9hvp \\
Cyclic urea & 1bv7, 1bv9, 1bwa, 1bwb, 1dmp, 1g35, 1hpo, 1mes, 1meu, 1pro, 1qbr, 1qbt, 1qbu, 7upj \\
\hline
\end{tabular}

Table 2

Parameter settings.

\begin{tabular}{ll}
\hline Common parameters & \\
\hline Swarm size & 150 Particles \\
Maximum number of evaluations & $1,000,000$ \\
& \\
SMPSO [9] \& SMPSOhv [29] \& SMPSOD \& SMPSOC \\
\hline Archive Size & 100 \\
$C_{1}, C_{2}$ & 1.5 \\
$\omega$ & 0.9 \\
Mutation & polynomial mutation \\
Mutation probability & 1.66 \\
Mutation distribution index $\eta_{m}$ & 20 \\
Selection method & Rounds \\
& \\
OMOPSO [6] & \\
\hline Archive size & 100 \\
$C_{1}, C_{2}$ & rand (1.5, 2.0) \\
$\omega$ & rand $(0.1,0.5)$ \\
Mutation & uniform + non-uniform + no mutation \\
Mutation probability & Each mutation is applied to $1 / 3$ of the swarm \\
& \\
MPSO/D [3] & \\
\hline$C_{1}, C_{2}$ & rand(1.5, 2.0) \\
$\omega$ & rand(0.1,0.5) \\
\hline
\end{tabular}

the obtained Pareto front approximations, although they use complementary calculation models from different reference points. It is worth noting that we are dealing with a real-world optimization problem, and therefore the true Pareto fronts to calculate the metrics are not known. To cope with this issue, we have generated a reference Pareto front for each instance by combining all the non-dominated solutions computed in all the executions of all the algorithms.

The computational framework used to deploy all the experiments consists in a Condor ${ }^{6}$ system, a middleware platform acting as the distributed task scheduler of up to 400 cores. This infrastructure is located at the Ada Byron Research Center at the University of Málaga (Spain), which comprises a set of IBM hosting racks for storage, units of virtualization, server compounds and backup services.

The parameter settings are summarized in Table 2 . We set a common subset of parameters that are the same for all the algorithms evaluated. The size of the swarm is 150 and the stopping condition is reached when 1,000,000 function evaluations are performed. These parameters have been chosen after preliminary tests where the stop condition and the swarm size were tuned to reach convergence enough (in accordance with reference Pareto front approximation) for most of algorithms. Similarly, the archive size is set to 100 , when applicable. All SMPSO versions use the polynomial mutation with distribution index $\eta_{m}=20$, which is applied to one sixth of the particles in the swarm. The acceleration coefficients $C_{1}$ and $C_{2}$ are set to 1.5 and the inertia weight is $\omega=0.9$. Those specific parameters to particle swarm algorithmic model where set as recommenced in Refs. [23] and [24],

\footnotetext{
${ }^{6}$ In URL: http://research.cs.wisc.edu/htcondor/.
}

where SMPSO obtained outstanding docking conformations in comparison with other prominent optimizers in the state of the art (NSGA-II, GDE3, and MOEA/D). Our approach is then to use common parameter setting to make the comparisons as fair as possible, but keeping the rest of the parameters as described in their original papers.

\section{Results and analysis}

In this section, we start by assessing the performance of the algorithms with statistical comparisons. Then, we carry out a further analysis with some selected solutions in terms of molecular conformations from a biological point of view.

\subsection{Performance comparisons}

Table 3 shows the median and interquartile range of the computed distributions (out of 31 independent runs) in terms of the Hypervolume indicator $I_{H V}$, for the set of 75 docking instances and for the 6 algorithms under comparison. This indicator measures convergence and diversity by computing the sum of the contributed volume of each point in the Pareto front (non-dominated solutions) with regards to a reference point. Therefore, the higher the convergence and diversity degree of a front, the higher (better) the resulting $I_{H V}$ value.

As we can observe in Table 3, MPSO/D obtains the best median values of $I_{H V}$ for a higher number of molecular instances (48 out of 75), followed by SMPSOhv with 26 best median values and $40 \mathrm{~s}$ best medians (best and second best results are shaded in dark and light grey, respectively). SMPSOD is the third best performing technique with best median value for one compound (1kzk) and second best median for 
Table 3

Median and interquartile range of the computed distributions (out of 31 independent runs) in terms of the hypervolume indicator $I_{H V}$, for the set of 75 docking instances and for the five compared algorithms: OMOPSO, MPSO/D SMPSO, SMPSOC, SMPSOD, and SMPSOhv.

\begin{tabular}{|c|c|c|c|c|c|c|c|}
\hline Molecular Category & Instance & OMOPSO & MPSO/D & SMPSO & SMPSOC & SMPSOD & SMPSOhv \\
\hline \multirow{22}{*}{ Small-size } & $1 \mathrm{a} 9 \mathrm{~m}$ & $0.00 e+00_{0.0 e+00}$ & $6.96 e-01_{1.2 e-01}$ & $6.44 e-01_{1.5 e-02}$ & $6.06 e-01_{2.8 e-02}$ & $6.60 e-01_{2.1 e-02}$ & $6.80 e-01_{6.4 e-03}$ \\
\hline & $1 \mathrm{aaq}$ & $0.00 e+00_{7.6 e-01}$ & $7.78 e-01_{1.8 e-02}$ & $7.28 e-01_{1.2 e-02}$ & $7.30 e-01_{1.1 e-02}$ & $7.35 e-01_{2.4 e-02}$ & $7.57 e-01_{2.5 e-02}$ \\
\hline & $1 \mathrm{~b} 61$ & $0.00 e+00_{1.5 e-01}$ & $6.45 e-01_{1.5 e-02}$ & $5.63 e-01_{2.6 e-02}$ & $4.69 e-01_{5.7 e-02}$ & $6.04 e-01_{1.8 e-02}$ & $6.21 e-01_{1.6 e-02}$ \\
\hline & $1 \mathrm{~b} 6 \mathrm{~m}$ & $0.00 e+00_{0.0 e+00}$ & $4.00 e-01_{4.5 e-01}$ & $3.52 e-01_{2.4 e-02}$ & $3.26 e-01_{5.6 e-02}$ & $3.60 e-01_{1.4 e-02}$ & $3.73 e-01_{1.2 e-02}$ \\
\hline & 1bdl & $6.01 e-01_{1.7 e-01}$ & $6.09 e-01_{3.7 e-03}$ & $5.49 e-01_{2.5 e-02}$ & $5.16 e-01_{3.0 e-02}$ & $5.67 e-01_{1.8 e-02}$ & $5.90 e-01_{1.1 e-02}$ \\
\hline & $1 \mathrm{bdq}$ & $0.00 e+00_{0.0 e+00}$ & $6.88 e-01_{3.5 e-02}$ & $5.72 e-01_{6.4 e-02}$ & $4.75 e-01_{8.9 e-02}$ & $5.95 e-01_{4.3 e-02}$ & $6.26 e-01_{3.7 e-02}$ \\
\hline & $1 \mathrm{bdr}$ & $0.00 e+00_{0.0 e+00}$ & $5.94 e-01_{1.6 e-01}$ & $4.85 e-01_{5.1 e-02}$ & $4.39 e-01_{4.4 e-02}$ & $4.82 e-01_{9.5 e-02}$ & $5.22 e-01_{5.7 e-02}$ \\
\hline & $1 \mathrm{gnm}$ & $0.00 e+00_{0.0 e+00}$ & $7.50 e-01_{1.4 e-01}$ & $6.84 e-01_{2.6 e-02}$ & $6.21 e-01_{5.8 e-02}$ & $7.13 e-01_{1.3 e-02}$ & $7.40 e-01_{7.4 e-03}$ \\
\hline & $1 \mathrm{gnn}$ & $0.00 e+00_{0.0 e+00}$ & $7.36 e-01_{4.4 e-01}$ & $6.82 e-01_{3.8 e-02}$ & $6.58 e-01_{2.5 e-02}$ & $7.03 e-01_{2.8 e-02}$ & $7.30 e-01_{2.1 e-02}$ \\
\hline & 1 gno & $0.00 e+00_{0.0 e+00}$ & $7.64 e-01_{3.6 e-01}$ & $7.68 e-01_{1.6 e-02}$ & $7.33 e-01_{3.0 e-02}$ & $7.82 e-01_{8.2 e-03}$ & $7.92 e-01_{1.1 e-02}$ \\
\hline & $1 \mathrm{hbv}$ & $0.00 e+00_{0.0 e+00}$ & $5.10 e-01_{7.2 e-02}$ & $3.89 e-01_{1.6 e-02}$ & $3.44 e-01_{4.1 e-02}$ & $4.01 e-01_{3.9 e-02}$ & $4.22 e-01_{7.3 e-03}$ \\
\hline & 1heg & $4.41 e-01_{3.7 e-01}$ & $6.02 e-01_{9.5 e-02}$ & $4.53 e-01_{5.7 e-02}$ & $4.08 e-01_{6.1 e-02}$ & $5.09 e-01_{8.5 e-02}$ & $4.81 e-01_{7.5 e-02}$ \\
\hline & 1hih & $0.00 e+00_{0.0 e+00}$ & $4.57 e-01_{1.2 e-01}$ & $3.96 e-01_{2.1 e-02}$ & $3.48 e-01_{2.8 e-02}$ & $4.16 e-01_{2.6 e-02}$ & $4.34 e-01_{1.6 e-02}$ \\
\hline & $1 \mathrm{hpv}$ & $0.00 e+00_{1.3 e-01}$ & $6.00 e-01_{3.4 e-01}$ & $5.78 e-01_{2.7 e-02}$ & $5.15 e-01_{5.8 e-02}$ & $5.93 e-01_{5.8 e-02}$ & $6.12 e-01_{1.1 e-02}$ \\
\hline & 1hsg & $0.00 e+00_{0.0 e+00}$ & $0.00 e+00_{6.5 e-01}$ & $5.32 e-01_{9.0 e-02}$ & $4.60 e-01_{7.2 e-02}$ & $5.59 e-01_{7.8 e-02}$ & $6.28 e-01_{6.7 e-02}$ \\
\hline & 1hte & $3.25 e-024.1 e-01$ & $4.35 e-01_{7.6 e-02}$ & $3.76 e-01_{2.5 e-02}$ & $3.35 e-01_{1.6 e-02}$ & $3.82 e-01_{1.5 e-02}$ & $3.95 e-01_{8.2 e-03}$ \\
\hline & $1 \mathrm{kzk}$ & $0.00 e+00_{0.0 e+00}$ & $3.25 e-01_{2.0 e-01}$ & $0.00 e+00_{0.0 e+00}$ & $0.00 e+00_{0.0 e+00}$ & $6.27 e-01_{8.3 e-02}$ & $0.00 e+00_{0.0 e+00}$ \\
\hline & $1 \mathrm{sbg}$ & $0.00 e+00_{0.0 e+00}$ & $4.87 e-01_{1.0 e-01}$ & $5.20 e-01_{2.7 e-02}$ & $4.75 e-01_{4.9 e-02}$ & $5.31 e-01_{2.7 e-02}$ & $5.55 e-01_{6.3 e-03}$ \\
\hline & 1 tcx & $0.00 e+00_{0.0 e+00}$ & $5.75 e-01_{1.2 e-01}$ & $5.29 e-01_{2.9 e-02}$ & $4.26 e-01_{1.5 e-01}$ & $5.53 e-01_{2.3 e-02}$ & $5.76 e-01_{5.2 e-03}$ \\
\hline & 1zih & $0.00 e+00_{0.0 e+00}$ & $6.73 e-01_{1.1 e-01}$ & $5.97 e-01_{3.9 e-02}$ & $5.43 e-01_{4.6 e-02}$ & $6.17 e-01_{4.8 e-02}$ & $6.56 e-01_{2.4 e-02}$ \\
\hline & 1zir & $0.00 e+00_{0.0 e+00}$ & $6.73 e-01_{1.9 e-01}$ & $5.69 e-01_{8.2 e-02}$ & $5.02 e-01_{4.6 e-02}$ & $5.71 e-01_{5.8 e-02}$ & $6.27 e-01_{4.0 e-02}$ \\
\hline & 3aid & $0.00 e+00_{0.0 e+00}$ & $5.36 e-01_{1.4 e-01}$ & $4.40 e-01_{4.0 e-02}$ & $4.02 e-01_{2.6 e-02}$ & $4.03 e-01_{9.3 e-02}$ & $4.44 e-01_{5.0 e-02}$ \\
\hline \multirow{22}{*}{ Medium-size } & $1 \mathrm{~b} 6 \mathrm{j}$ & $0.00 e+00_{0.0 e+00}$ & $6.51 e-01_{5.7 e-02}$ & $5.22 e-01_{4.3 e-02}$ & $4.72 e-01_{2.6 e-02}$ & $5.53 e-01_{4.9 e-02}$ & $6.07 e-01_{3.9 e-02}$ \\
\hline & $1 \mathrm{~b} 6 \mathrm{k}$ & $0.00 e+00_{0.0 e+00}$ & $4.61 e-01_{4.1 e-01}$ & $4.11 e-01_{1.5 e-02}$ & $3.89 e-01_{2.0 e-02}$ & $4.18 e-01_{1.4 e-02}$ & $4.38 e-01_{7.2 e-03}$ \\
\hline & $1 \mathrm{~b} 6 \mathrm{p}$ & $0.00 e+00_{0.0 e+00}$ & $0.00 e+00_{6.2 e-01}$ & $5.27 e-01_{2.0 e-02}$ & $5.08 e-01_{5.5 e-02}$ & $5.79 e-01_{2.2 e-02}$ & $5.87 e-01_{1.8 e-02}$ \\
\hline & $1 \mathrm{~d} 4 \mathrm{k}$ & $0.00 e+00_{0.0 e+00}$ & $0.00 e+00_{5.7 e-01}$ & $5.90 e-01_{2.6 e-02}$ & $5.59 e-01_{4.1 e-02}$ & $6.23 e-01_{1.5 e-02}$ & $6.39 e-01_{1.4 e-02}$ \\
\hline & $1 \mathrm{~d} 41$ & $0.00 e+00_{0.0 e+00}$ & $0.00 e+00_{8.0 e-01}$ & $7.66 e-01_{1.4 e-02}$ & $7.55 e-01_{1.2 e-02}$ & $7.79 e-01_{5.9 e-03}$ & $7.91 e-01_{1.9 e-03}$ \\
\hline & 1hef & $8.80 e-01_{4.0 e-02}$ & $8.94 e-01_{4.6 e-03}$ & $8.39 e-01_{1.9 e-02}$ & $8.47 e-01_{1.3 e-02}$ & $8.72 e-01_{3.4 e-03}$ & $8.75 e-01_{5.2 e-03}$ \\
\hline & $1 \mathrm{hps}$ & $0.00 e+00_{0.0 e+00}$ & $7.87 e-01_{8.2 e-01}$ & $8.39 e-01_{1.2 e-02}$ & $8.30 e-01_{7.0 e-03}$ & $8.33 e-01_{1.1 e-02}$ & $8.58 e-01_{1.4 e-02}$ \\
\hline & $1 \mathrm{hxw}$ & $4.81 e-01_{5.0 e-01}$ & $5.21 e-01_{3.7 e-02}$ & $4.59 e-01_{4.2 e-02}$ & $4.43 e-01_{3.0 e-02}$ & $4.70 e-01_{3.4 e-02}$ & $4.83 e-01_{3.1 e-02}$ \\
\hline & lizh & $0.00 e+00_{6.6 e-02}$ & $7.32 e-01_{4.3 e-01}$ & $7.24 e-01_{1.5 e-02}$ & $6.96 e-01_{2.0 e-02}$ & $7.22 e-01_{1.4 e-02}$ & $7.50 e-01_{4.6 e-03}$ \\
\hline & lizi & $0.00 e+00_{3.6 e-01}$ & $7.19 e-01_{1.1 e-01}$ & $6.23 e-01_{1.7 e-01}$ & $4.65 e-01_{1.2 e-01}$ & $6.63 e-01_{2.1 e-01}$ & $7.04 e-01_{1.9 e-01}$ \\
\hline & 1jld & $6.06 e-01_{6.2 e-01}$ & $6.19 e-01_{8.4 e-02}$ & $5.56 e-01_{2.3 e-02}$ & $4.97 e-01_{6.3 e-02}$ & $5.73 e-01_{5.8 e-02}$ & $6.10 e-01_{1.1 e-02}$ \\
\hline & $1 \mathrm{k} 6 \mathrm{c}$ & $0.00 e+00_{0.0 e+00}$ & $3.71 e-01_{5.9 e-01}$ & $5.25 e-01_{3.4 e-02}$ & $4.96 e-01_{5.4 e-02}$ & $5.51 e-01_{1.8 e-02}$ & $5.76 e-01_{1.1 e-02}$ \\
\hline & $1 \mathrm{k} 6 \mathrm{t}$ & $0.00 e+00_{0.0 e+00}$ & $5.29 e-01_{5.4 e-01}$ & $4.14 e-01_{4.2 e-02}$ & $3.94 e-01_{1.8 e-02}$ & $4.52 e-01_{5.8 e-02}$ & $5.14 e-01_{7.0 e-02}$ \\
\hline & $1 \mathrm{k} 6 \mathrm{p}$ & $0.00 e+00_{0.0 e+00}$ & $0.00 e+00_{5.1 e-01}$ & $3.67 e-01_{7.7 e-02}$ & $3.19 e-01_{4.1 e-02}$ & $3.80 e-01_{7.8 e-02}$ & $4.64 e-01_{6.8 e-02}$ \\
\hline & $1 \mathrm{k} 6 \mathrm{v}$ & $0.00 e+00_{0.0 e+00}$ & $0.00 e+00_{5.8 e-01}$ & $5.07 e-01_{2.7 e-02}$ & $4.71 e-01_{4.6 e-02}$ & $5.08 e-01_{3.7 e-02}$ & $5.66 e-01_{1.8 e-02}$ \\
\hline & $1 \mathrm{mtr}$ & $0.00 e+00_{0.0 e+00}$ & $5.00 e-01_{5.5 e-01}$ & $4.35 e-01_{6.1 e-02}$ & $3.93 e-01_{5.2 e-02}$ & $4.76 e-01_{4.1 e-02}$ & $5.05 e-01_{3.0 e-02}$ \\
\hline & $1 \mathrm{mui}$ & $0.00 e+00_{0.0 e+00}$ & $6.59 e-01_{5.7 e-01}$ & $6.21 e-01_{2.0 e-02}$ & $6.18 e-01_{2.5 e-02}$ & $6.41 e-01_{6.5 e-03}$ & $6.57 e-01_{7.3 e-03}$ \\
\hline & $2 \mathrm{bpv}$ & $4.98 e-01_{6.8 e-02}$ & $5.61 e-01_{5.0 e-02}$ & $3.45 e-01_{2.5 e-02}$ & $3.12 e-01_{2.4 e-02}$ & $3.66 e-01_{2.4 e-02}$ & $3.82 e-01_{4.5 e-02}$ \\
\hline & $2 b p x$ & $0.00 e+00_{0.0 e+00}$ & $2.60 e-01_{6.1 e-01}$ & $4.97 e-01_{4.8 e-02}$ & $4.56 e-01_{4.4 e-02}$ & $5.81 e-01_{7.9 e-02}$ & $5.96 e-01_{2.7 e-02}$ \\
\hline & 4 hvp & $0.00 e+00_{0.0 e+00}$ & $6.86 e-01_{8.6 e-03}$ & $6.28 e-01_{2.0 e-02}$ & $5.64 e-01_{7.4 e-02}$ & $6.41 e-01_{2.1 e-02}$ & $6.64 e-01_{1.4 e-02}$ \\
\hline & 4phv & $0.00 e+00_{0.0 e+00}$ & $6.39 e-01_{6.4 e-01}$ & $6.12 e-01_{1.2 e-02}$ & $5.82 e-01_{1.7 e-02}$ & $6.22 e-01_{2.1 e-01}$ & $6.40 e-01_{2.3 e-03}$ \\
\hline & 5 hvp & $0.00 e+00_{0.0 e+00}$ & $8.63 e-01_{2.2 e-01}$ & $7.44 e-01_{6.6 e-02}$ & $7.09 e-01_{2.3 e-02}$ & $7.62 e-01_{1.0 e-01}$ & $8.25 e-01_{4.7 e-02}$ \\
\hline \multirow{17}{*}{ Large -size } & 1a94 & $3.55 e-01_{4.6 e-01}$ & $4.04 e-01_{8.7 e-02}$ & $4.03 e-01_{2.2 e-02}$ & $3.76 e-01_{3.3 e-02}$ & $4.34 e-01_{3.2 e-02}$ & $4.53 e-01_{1.1 e-02}$ \\
\hline & 1hiv & $0.00 e+00_{0.0 e+00}$ & $5.73 e-01_{3.4 e-01}$ & $5.84 e-01_{3.8 e-02}$ & $5.53 e-01_{3.3 e-02}$ & $6.10 e-01_{1.4 e-02}$ & $6.29 e-01_{3.4 e-02}$ \\
\hline & 1hos & $3.99 e-01_{3.5 e-02}$ & $4.30 e-01_{4.8 e-02}$ & $3.45 e-01_{2.9 e-02}$ & $2.88 e-01_{3.1 e-02}$ & $3.83 e-01_{1.6 e-02}$ & $3.90 e-01_{1.2 e-02}$ \\
\hline & lhtg & $0.00 e+00_{0.0 e+00}$ & $5.95 e-01_{4.8 e-01}$ & $3.98 e-01_{2.8 e-02}$ & $3.71 e-01_{2.0 e-02}$ & $3.96 e-01_{2.2 e-02}$ & $4.21 e-01_{5.3 e-02}$ \\
\hline & lhvi & $0.00 e+00_{0.0 e+00}$ & $7.04 e-01_{1.9 e-02}$ & $6.58 e-01_{2.1 e-02}$ & $6.24 e-01_{1.7 e-02}$ & $6.83 e-01_{2.0 e-02}$ & $6.90 e-01_{3.0 e-02}$ \\
\hline & $1 \mathrm{hvj}$ & $0.00 e+00_{0.0 e+00}$ & $6.38 e-01_{2.6 e-03}$ & $5.92 e-01_{1.6 e-02}$ & $5.67 e-01_{1.3 e-02}$ & $6.18 e-01_{1.8 e-02}$ & $6.30 e-01_{3.0 e-02}$ \\
\hline & $1 \mathrm{hvk}$ & $0.00 e+00_{0.0 e+00}$ & $3.83 e-01_{2.4 e-01}$ & $5.72 e-01_{1.3 e-02}$ & $5.47 e-01_{2.1 e-02}$ & $5.97 e-01_{2.9 e-03}$ & $6.05 e-01_{4.2 e-03}$ \\
\hline & lhvl & $0.00 e+00_{0.0 e+00}$ & $6.16 e-01_{2.7 e-01}$ & $5.62 e-01_{1.4 e-02}$ & $5.36 e-01_{1.5 e-02}$ & $5.95 e-01_{1.5 e-02}$ & $6.01 e-01_{1.2 e-02}$ \\
\hline & 1hvs & $0.00 e+00_{0.0 e+00}$ & $6.54 e-01_{1.6 e-01}$ & $6.22 e-01_{1.5 e-02}$ & $5.88 e-01_{1.8 e-02}$ & $6.43 e-01_{8.3 e-03}$ & $6.61 e-01_{1.0 e-02}$ \\
\hline & 1hwr & $0.00 e+00_{5.7 e-01}$ & $5.74 e-01_{2.6 e-03}$ & $5.34 e-01_{2.0 e-02}$ & $5.16 e-01_{2.6 e-02}$ & $5.51 e-01_{1.4 e-02}$ & $5.67 e-01_{5.0 e-03}$ \\
\hline & lody & $0.00 e+00_{0.0 e+00}$ & $5.38 e-01_{4.2 e-01}$ & $6.79 e-01_{1.3 e-02}$ & $6.57 e-01_{1.8 e-02}$ & $7.02 e-01_{3.4 e-02}$ & $7.21 e-01_{3.1 e-02}$ \\
\hline & $1 \mathrm{vij}$ & $0.00 e+00_{0.0 e+00}$ & $5.54 e-01_{6.8 e-02}$ & $4.71 e-01_{5.0 e-02}$ & $4.39 e-01_{2.6 e-02}$ & $5.20 e-01_{6.9 e-02}$ & $5.23 e-01_{6.3 e-02}$ \\
\hline & 1vik & $0.00 e+00_{0.0 e+00}$ & $6.52 e-01_{2.6 e-02}$ & $5.97 e-01_{9.9 e-03}$ & $5.69 e-01_{2.8 e-02}$ & $6.09 e-01_{2.0 e-02}$ & $6.23 e-019.5 e-03$ \\
\hline & 3 tlh & $1.53 e-01_{5.0 e-01}$ & $5.15 e-01_{1.2 e-01}$ & $4.68 e-01_{2.2 e-02}$ & $4.42 e-01_{1.4 e-02}$ & $4.55 e-01_{2.7 e-02}$ & $4.84 e-01_{2.8 e-02}$ \\
\hline & 7 hvp & $6.35 e-01_{1.3 e-01}$ & $7.25 e-01_{3.1 e-02}$ & $6.05 e-01_{3.3 e-02}$ & $5.84 e-01_{1.9 e-02}$ & $6.04 e-01_{3.1 e-02}$ & $6.19 e-01_{5.2 e-02}$ \\
\hline & 8 hvp & $3.84 e-01_{6.7 e-02}$ & $4.32 e-01_{5.0 e-02}$ & $2.51 e-01_{3.4 e-02}$ & $2.48 e-01_{2.6 e-02}$ & $3.32 e-01_{3.8 e-02}$ & $3.33 e-01_{3.9 e-02}$ \\
\hline & 9 hvp & $0.00 e+00_{4.3 e-01}$ & $4.06 e-01_{1.7 e-01}$ & $3.83 e-01_{4.6 e-02}$ & $3.13 e-01_{5.0 e-02}$ & $4.02 e-01_{3.3 e-02}$ & $4.30 e-01_{1.4 e-02}$ \\
\hline \multirow{14}{*}{ Cyclic-urea } & $1 \mathrm{bv} 7$ & $0.00 e+00_{0.0 e+00}$ & $5.98 e-01_{5.6 e-02}$ & $4.08 e-01_{1.0 e-01}$ & $3.43 e-01_{5.9 e-02}$ & $3.81 e-01_{9.9 e-02}$ & $5.02 e-01_{7.7 e-02}$ \\
\hline & 1bv9 & $0.00 e+00_{0.0 e+00}$ & $6.02 e-01_{8.0 e-02}$ & $3.74 e-01_{5.0 e-02}$ & $3.43 e-01_{5.1 e-02}$ & $4.08 e-01_{7.1 e-02}$ & $4.60 e-01_{9.5 e-02}$ \\
\hline & 1bwa & $0.00 e+00_{0.0 e+00}$ & $6.41 e-01_{4.6 e-02}$ & $4.90 e-01_{6.6 e-02}$ & $4.49 e-01_{3.9 e-02}$ & $5.37 e-01_{6.9 e-02}$ & $5.57 e-01_{6.1 e-02}$ \\
\hline & $1 \mathrm{bwb}$ & $0.00 e+00_{0.0 e+00}$ & $6.83 e-01_{5.6 e-02}$ & $6.45 e-01_{3.6 e-02}$ & $5.90 e-01_{4.2 e-02}$ & $6.61 e-01_{3.6 e-02}$ & $6.88 e-01_{1.8 e-02}$ \\
\hline & $1 \mathrm{dmp}$ & $0.00 e+00_{0.0 e+00}$ & $5.91 e-01_{3.1 e-02}$ & $5.18 e-01_{1.3 e-02}$ & $5.03 e-01_{8.7 e-03}$ & $5.29 e-01_{1.3 e-02}$ & $5.38 e-01_{3.2 e-02}$ \\
\hline & $1 \mathrm{~g} 35$ & $0.00 e+00_{0.0 e+00}$ & $4.90 e-01_{4.8 e-01}$ & $5.72 e-01_{2.8 e-02}$ & $5.46 e-01_{2.4 e-02}$ & $5.84 e-01_{2.5 e-02}$ & $6.04 e-01_{2.0 e-02}$ \\
\hline & 1 hpo & $0.00 e+00_{0.0 e+00}$ & $7.17 e-01_{7.2 e-01}$ & $6.65 e-01_{2.0 e-02}$ & $6.07 e-01_{4.6 e-02}$ & $6.78 e-01_{1.9 e-02}$ & $7.00 e-01_{1.0 e-02}$ \\
\hline & $1 \mathrm{mes}$ & $0.00 e+00_{0.0 e+00}$ & $6.12 e-01_{1.9 e-01}$ & $5.55 e-01_{8.6 e-02}$ & $5.33 e-01_{8.0 e-02}$ & $5.71 e-01_{6.9 e-02}$ & $6.30 e-01_{4.6 e-02}$ \\
\hline & $1 \mathrm{meu}$ & $0.00 e+00_{0.0 e+00}$ & $5.24 e-01_{9.8 e-02}$ & $4.94 e-01_{5.0 e-02}$ & $4.58 e-01_{8.7 e-02}$ & $5.12 e-01_{6.1 e-02}$ & $5.41 e-01_{1.2 e-02}$ \\
\hline & 1 pro & $0.00 e+00_{0.0 e+00}$ & $6.81 e-01_{1.0 e-01}$ & $6.00 e-01_{5.5 e-02}$ & $5.82 e-01_{3.1 e-02}$ & $5.71 e-01_{5.5 e-02}$ & $6.40 e-01_{9.3 e-02}$ \\
\hline & $1 \mathrm{qbr}$ & $0.00 e+00_{0.0 e+00}$ & $5.99 e-01_{6.1 e-01}$ & $4.78 e-01_{7.8 e-02}$ & $4.03 e-01_{7.2 e-02}$ & $4.92 e-01_{8.0 e-02}$ & $5.24 e-01_{5.0 e-02}$ \\
\hline & $1 \mathrm{qbt}$ & $0.00 e+00_{0.0 e+00}$ & $6.33 e-01_{1.9 e-01}$ & $4.76 e-01_{6.1 e-02}$ & $4.04 e-01_{6.5 e-02}$ & $4.93 e-01_{4.5 e-02}$ & $5.52 e-01_{4.5 e-02}$ \\
\hline & $1 \mathrm{qbu}$ & $0.00 e+00_{0.0 e+00}$ & $5.12 e-01_{5.6 e-01}$ & $4.49 e-01_{3.1 e-02}$ & $4.08 e-01_{3.9 e-02}$ & $4.16 e-01_{6.4 e-02}$ & $4.75 e-01_{5.8 e-02}$ \\
\hline & $7 \mathrm{upj}$ & $1.55 e-02_{6.8 e-01}$ & $7.10 e-01_{5.3 e-02}$ & $6.37 e-01_{3.4 e-02}$ & $6.05 e-01_{3.7 e-02}$ & $6.33 e-01_{3.0 e-02}$ & $6.62 e-01_{3.8 e-02}$ \\
\hline
\end{tabular}


Table 4

Median and interquartile range of the computed distributions (out of 31 independent runs) in terms of the Inverted Generational Instance Plus indicator $I_{I G D+}$, for the set of 75 docking instances and for the five compared algorithms: OMOPSO, MPSO/D, SMPSO, SMPSOC, SMPSOD, and SMPSOhv.

\begin{tabular}{|c|c|c|c|c|c|c|c|}
\hline Molecular Category & Instance & OMOPSO & MPSO/D & SMPSO & SMPSOC & SMPSOD & SMPSOhv \\
\hline \multirow{22}{*}{ Small-size } & $1 \mathrm{a} 9 \mathrm{~m}$ & $1.73 e-01_{3.5 e+00}$ & $1.28 e-01_{8.7 e-02}$ & $7.50 e-02_{1.2 e-02}$ & $8.80 e-02_{1.9 e-02}$ & $5.86 e-02_{1.6 e-02}$ & $4.79 e-02_{5.9 e-03}$ \\
\hline & $1 \mathrm{aaq}$ & $2.73 e+00_{7.0 e+00}$ & $1.09 e-029.7 e-03$ & $4.18 e-02_{7.0 e-03}$ & $3.87 e-02_{7.0 e-03}$ & $3.74 e-02_{1.2 e-02}$ & $2.53 e-02_{1.3 e-02}$ \\
\hline & $1 \mathrm{~b} 61$ & $2.67 e+00_{3.1 e+00}$ & $5.66 e-03_{7.5 e-03}$ & $4.93 e-02_{1.4 e-02}$ & $1.09 e-01_{4.0 e-02}$ & $2.55 e-029.9 e-03$ & $1.65 e-028.1 e-03$ \\
\hline & $1 \mathrm{~b} 6 \mathrm{~m}$ & $2.45 e+00_{1.2 e+00}$ & $4.92 e-023.7 e-01$ & $6.85 e-02_{1.1 e-02}$ & $7.63 e-02_{2.4 e-02}$ & $6.39 e-02_{4.6 e-03}$ & $5.66 e-02_{4.0 e-03}$ \\
\hline & 1bdl & $8.56 e-03_{2.7 e-01}$ & $5.69 e-03_{1.3 e-03}$ & $3.07 e-02_{1.2 e-02}$ & $4.49 e-02_{1.7 e-02}$ & $2.30 e-02_{1.1 e-02}$ & $9.60 e-03_{3.6 e-03}$ \\
\hline & $1 \mathrm{bdq}$ & $8.39 e+00_{3.5 e+00}$ & $1.35 e-02_{1.7 e-02}$ & $4.71 e-02_{2.5 e-02}$ & $8.37 e-023.9 e-02$ & $3.64 e-02_{1.4 e-02}$ & $2.39 e-029.1 e-03$ \\
\hline & $1 \mathrm{bdr}$ & $1.34 e+01_{6.2 e+00}$ & $3.06 e-02_{1.1 e-01}$ & $7.45 e-02_{2.8 e-02}$ & $1.03 e-01_{2.8 e-02}$ & $7.93 e-025.8 e-02$ & $5.32 e-02_{3.4 e-02}$ \\
\hline & $1 \mathrm{gnm}$ & $6.44 e+00_{4.9 e-01}$ & $9.49 e-03_{1.1 e-01}$ & $4.91 e-02_{1.7 e-02}$ & $9.25 e-02_{4.2 e-02}$ & $3.03 e-02_{8.6 e-03}$ & $1.50 e-02_{4.1 e-03}$ \\
\hline & $1 \mathrm{gnn}$ & $8.13 e+00_{7.1 e-01}$ & $8.54 e-03_{3.3 e-01}$ & $4.62 e-02_{2.9 e-02}$ & $6.66 e-02_{2.2 e-02}$ & $2.88 e-02_{2.3 e-02}$ & $1.32 e-02_{1.5 e-02}$ \\
\hline & 1gno & $5.49 e+00_{5.9 e-01}$ & $3.76 e-02_{2.7 e-01}$ & $2.56 e-02_{8.1 e-03}$ & $4.43 e-02_{1.7 e-02}$ & $1.87 e-023.8 e-03$ & $1.30 e-024.7 e-03$ \\
\hline & $1 \mathrm{hbv}$ & $1.51 e+00_{2.9 e+00}$ & $2.46 e-023.5 e-02$ & $7.95 e-02_{7.1 e-03}$ & $1.01 e-01_{2.2 e-02}$ & $7.30 e-02_{2.2 e-02}$ & $6.02 e-02_{3.4 e-03}$ \\
\hline & 1heg & $1.53 e-01_{3.8 e-01}$ & $2.70 e-023.5 e-02$ & $9.69 e-02_{3.3 e-02}$ & $1.19 e-01_{3.7 e-02}$ & $6.07 e-024.1 e-02$ & $7.98 e-02_{4.3 e-02}$ \\
\hline & 1hih & $3.32 e+00_{3.0 e+00}$ & $1.95 e-02_{1.2 e-01}$ & $3.86 e-02_{5.0 e-03}$ & $5.28 e-02_{1.3 e-02}$ & $3.02 e-025.4 e-03$ & $2.42 e-023.6 e-03$ \\
\hline & $1 \mathrm{hpv}$ & $4.66 e+00_{5.1 e+00}$ & $6.11 e-02_{3.2 e-01}$ & $3.35 e-02_{9.9 e-03}$ & $5.25 e-02_{2.4 e-02}$ & $2.68 e-02_{1.8 e-02}$ & $1.86 e-02_{3.6 e-03}$ \\
\hline & 1hsg & $8.21 e+00_{1.5 e+00}$ & $2.41 e+00_{4.3 e+00}$ & $6.52 e-02_{4.1 e-02}$ & $9.76 e-022_{3.7 e-02}$ & $4.97 e-023.4 e-02$ & $1.92 e-022.6 e-02$ \\
\hline & 1hte & $6.42 e-01_{8.8 e-01}$ & $3.22 e-02_{2.2 e-02}$ & $5.00 e-02_{1.1 e-02}$ & $6.35 e-024.2 e-03$ & $4.69 e-02_{6.0 e-03}$ & $4.02 e-02_{2.1 e-03}$ \\
\hline & $1 \mathrm{kzk}$ & $3.14 e-01_{1.6 e-01}$ & $3.61 e-02_{4.2 e-02}$ & $2.93 e-01_{4.0 e-02}$ & $2.92 e-01_{1.0 e-01}$ & $1.72 e-02_{1.1 e-02}$ & $3.11 e-01_{2.0 e-01}$ \\
\hline & $1 \mathrm{sbg}$ & $1.02 e+01_{8.2 e+00}$ & $6.65 e-022_{8.3 e-02}$ & $2.80 e-02_{1.3 e-02}$ & $5.20 e-02_{2.9 e-02}$ & $2.24 e-02_{1.3 e-02}$ & $1.01 e-02_{2.6 e-03}$ \\
\hline & $1 \mathrm{tcx}$ & $1.00 e+01_{5.2 e+00}$ & $9.80 e-03_{8.4 e-02}$ & $3.28 e-02_{1.6 e-02}$ & $9.96 e-02_{1.1 e-01}$ & $1.93 e-02_{1.0 e-02}$ & $7.00 e-03_{1.8 e-03}$ \\
\hline & 1zih & $1.59 e+01_{6.9 e+00}$ & $9.22 e-03_{5.7 e-02}$ & $4.40 e-02_{1.6 e-02}$ & $6.79 e-02_{2.5 e-02}$ & $3.41 e-02_{1.8 e-02}$ & $1.38 e-02_{8.9 e-03}$ \\
\hline & 1zir & $8.29 e+00_{1.0 e+01}$ & $8.06 e-03_{4.1 e-01}$ & $5.10 e-02_{3.6 e-02}$ & $7.68 e-02_{2.3 e-02}$ & $4.96 e-02_{2.3 e-02}$ & $2.17 e-02_{1.5 e-02}$ \\
\hline & 3aid & $4.99 e+00_{2.3 e+00}$ & $3.65 e-02_{1.0 e-01}$ & $7.35 e-02_{1.9 e-02}$ & $9.32 e-02_{1.5 e-02}$ & $9.16 e-025.9 e-02$ & $6.81 e-02_{2.3 e-02}$ \\
\hline \multirow{22}{*}{ Medium-size } & $1 \mathrm{~b} 6 \mathrm{j}$ & $9.66 e+00_{3.6 e+00}$ & $5.15 e-03.9 e-02$ & $7.52 e-02_{2.9 e-02}$ & $1.01 e-01_{1.8 e-02}$ & $5.37 e-02_{2.6 e-02}$ & $2.67 e-02_{1.9 e-02}$ \\
\hline & $1 \mathrm{~b} 6 \mathrm{k}$ & $5.97 e+00_{3.5 e+00}$ & $2.49 e-02_{5.0 e-01}$ & $4.49 e-02_{8.8 e-03}$ & $5.16 e-029.1 e-03$ & $3.98 e-024.8 e-03$ & $2.96 e-02_{2.7 e-03}$ \\
\hline & $1 \mathrm{~b} 6 \mathrm{p}$ & $6.80 e+00_{9.5 e-01}$ & $3.00 e+00_{4.3 e+00}$ & $5.47 e-02_{1.1 e-02}$ & $6.65 e-02_{3.5 e-02}$ & $2.79 e-02_{1.1 e-02}$ & $2.06 e-02_{8.6 e-03}$ \\
\hline & $1 \mathrm{~d} 4 \mathrm{k}$ & $1.09 e+01_{3.5 e+00}$ & $5.41 e+00_{7.5 e+00}$ & $5.71 e-02_{1.3 e-02}$ & $6.99 e-02_{1.9 e-02}$ & $4.35 e-02_{6.2 e-03}$ & $3.28 e-02_{5.9 e-03}$ \\
\hline & $1 \mathrm{~d} 41$ & $4.76 e+00_{3.6 e+00}$ & $1.99 e+00_{2.7 e+00}$ & $9.93 e-03_{3.8 e-03}$ & $1.09 e-02_{2.9 e-03}$ & $8.43 e-03_{1.8 e-03}$ & $3.01 e-03_{5.9 e-04}$ \\
\hline & 1hef & $5.41 e-03_{1.0 e-02}$ & $3.97 e-03_{8.8 e-04}$ & $1.74 e-02_{3.3 e-03}$ & $1.49 e-02_{3.0 e-03}$ & $1.15 e-02_{2.0 e-03}$ & $8.75 e-03_{3.6 e-03}$ \\
\hline & $1 \mathrm{hps}$ & $6.53 e+00_{4.5 e-01}$ & $5.90 e-022.8 e+00$ & $2.10 e-025.9 e-03$ & $2.39 e-02_{3.8 e-03}$ & $2.45 e-025.4 e-03$ & $1.05 e-02_{6.3 e-03}$ \\
\hline & 1hxw & $6.07 e-023.9 e+00$ & $3.18 e-02_{2.0 e-02}$ & $5.79 e-02_{1.6 e-02}$ & $6.26 e-02_{1.3 e-02}$ & $5.34 e-02_{1.2 e-02}$ & $4.48 e-02_{1.0 e-02}$ \\
\hline & lizh & $8.73 e+00_{5.3 e+00}$ & $2.32 e-02_{3.6 e-01}$ & $1.78 e-02_{6.8 e-03}$ & $2.76 e-02_{8.9 e-03}$ & $1.89 e-025.3 e-03$ & $5.02 e-03_{1.9 e-03}$ \\
\hline & 1izi & $3.28 e+00_{7.6 e+00}$ & $1.66 e-025.4 e-02$ & $4.46 e-027.1 e-02$ & $1.17 e-01_{6.5 e-02}$ & $2.66 e-02_{9.1 e-02}$ & $1.35 e-02_{6.9 e-02}$ \\
\hline & 1jld & $1.77 e-02_{6.9 e+00}$ & $1.04 e-027.8 e-02$ & $4.15 e-02_{1.2 e-02}$ & $7.64 e-02_{3.9 e-02}$ & $3.21 e-02_{3.3 e-02}$ & $1.15 e-02_{5.2 e-03}$ \\
\hline & $1 \mathrm{k} 6 \mathrm{c}$ & $6.48 e+00_{3.4 e+00}$ & $2.27 e-01_{2.4 e+00}$ & $3.57 e-02_{1.9 e-02}$ & $4.99 e-02_{3.1 e-02}$ & $2.31 e-029.4 e-03$ & $9.65 e-03_{4.8 e-03}$ \\
\hline & $1 \mathrm{k} 6 \mathrm{t}$ & $4.02 e+00_{1.1 e+00}$ & $1.59 e-029.8 e-01$ & $7.90 e-02_{2.7 e-02}$ & $9.19 e-02_{1.4 e-02}$ & $5.60 e-023.6 e-02$ & $1.80 e-02_{3.9 e-02}$ \\
\hline & $1 \mathrm{k} 6 \mathrm{p}$ & $4.34 e+00_{1.2 e+00}$ & $1.28 e+00_{3.6 e+00}$ & $9.98 e-022_{5.4 e-02}$ & $1.32 e-01_{3.0 e-02}$ & $9.08 e-025.1 e-02$ & $3.88 e-02_{3.9 e-02}$ \\
\hline & $1 \mathrm{k} 6 \mathrm{v}$ & $5.56 e+00_{1.7 e+00}$ & $1.90 e+00_{3.2 e+00}$ & $4.41 e-02_{1.5 e-02}$ & $6.12 e-02_{2.4 e-02}$ & $4.45 e-02_{1.8 e-02}$ & $1.65 e-027.7 e-03$ \\
\hline & $1 \mathrm{mtr}$ & $2.63 e+00_{1.4 e+00}$ & $5.80 e-028.2 e-01$ & $5.91 e-02_{2.2 e-02}$ & $7.25 e-02_{2.2 e-02}$ & $4.27 e-02_{1.4 e-02}$ & $3.36 e-029.3 e-03$ \\
\hline & $1 \mathrm{mui}$ & $1.01 e+01_{4.6 e+00}$ & $9.67 e-03_{5.9 e-01}$ & $2.40 e-029.6 e-03$ & $2.22 e-02_{1.3 e-02}$ & $1.49 e-023.7 e-03$ & $5.51 e-03_{1.8 e-03}$ \\
\hline & $2 \mathrm{bpv}$ & $5.21 e-02_{4.1 e-02}$ & $1.99 e-024.8 e-03$ & $7.59 e-02_{1.1 e-02}$ & $8.25 e-02_{8.3 e-03}$ & $6.22 e-02_{6.2 e-03}$ & $5.73 e-02_{1.1 e-02}$ \\
\hline & $2 \mathrm{bpx}$ & $8.79 e+00_{3.8 e+00}$ & $1.01 e+00_{4.1 e+00}$ & $7.25 e-02_{2.9 e-02}$ & $9.84 e-02_{2.9 e-02}$ & $2.14 e-024.7 e-02$ & $1.26 e-02_{1.4 e-02}$ \\
\hline & 4hvp & $6.84 e+00_{1.6 e+00}$ & $6.19 e-03_{1.2 e-03}$ & $2.82 e-029.7 e-03$ & $5.60 e-023.7 e-02$ & $2.30 e-027.4 e-03$ & $1.10 e-02_{4.5 e-03}$ \\
\hline & $4 \mathrm{phv}$ & $2.36 e+01_{7.8 e+00}$ & $5.08 e-03_{1.7 e+01}$ & $1.77 e-02_{6.3 e-03}$ & $2.94 e-02_{8.8 e-03}$ & $1.39 e-02_{1.3 e-01}$ & $3.40 e-03_{7.4 e-04}$ \\
\hline & 5 hvp & $2.99 e+00_{2.3 e+00}$ & $9.46 e-03_{1.5 e-01}$ & $5.35 e-02_{3.3 e-02}$ & $6.78 e-02_{1.2 e-02}$ & $4.11 e-024.7 e-02$ & $1.48 e-02_{1.9 e-02}$ \\
\hline \multirow{17}{*}{ Large -size } & $1 \mathrm{a} 94$ & $1.03 e+01_{1.4 e+00}$ & $1.46 e-02_{1.1 e-01}$ & $3.55 e-02_{7.3 e-03}$ & $5.14 e-02_{1.4 e-02}$ & $2.81 e-02_{1.0 e-02}$ & $1.59 e-02_{3.4 e-03}$ \\
\hline & 1hiv & $4.94 e+00_{3.8 e+00}$ & $5.42 e-022_{3.2 e-01}$ & $3.95 e-02_{1.8 e-02}$ & $5.48 e-02_{1.7 e-02}$ & $2.70 e-025.4 e-03$ & $1.70 e-02_{1.5 e-02}$ \\
\hline & 1hos & $4.02 e-02_{1.4 e-02}$ & $2.88 e-02_{1.3 e-02}$ & $5.11 e-02_{8.9 e-03}$ & $6.69 e-02_{1.1 e-02}$ & $3.86 e-023.9 e-03$ & $3.69 e-02_{3.9 e-03}$ \\
\hline & 1htg & $9.41 e+00_{1.9 e+00}$ & $1.94 e-024.7 e-01$ & $9.71 e-02_{1.6 e-02}$ & $1.12 e-01_{1.2 e-02}$ & $9.75 e-02_{1.2 e-02}$ & $8.32 e-02_{2.7 e-02}$ \\
\hline & 1hvi & $1.15 e+01_{2.7 e+00}$ & $1.44 e-02_{1.5 e-02}$ & $2.28 e-02_{6.6 e-03}$ & $3.50 e-022_{5.7 e-03}$ & $1.46 e-02_{8.2 e-03}$ & $9.52 e-03_{8.7 e-03}$ \\
\hline & $1 \mathrm{hvj}$ & $9.07 e+00_{3.4 e+00}$ & $7.67 e-03_{2.2 e-03}$ & $2.33 e-02_{6.4 e-03}$ & $3.17 e-024.7 e-03$ & $1.46 e-025.2 e-03$ & $6.78 e-039.2 e-03$ \\
\hline & $1 \mathrm{hvk}$ & $1.01 e+01_{9.8 e-01}$ & $2.27 e-01_{2.3 e-01}$ & $2.66 e-025.6 e-03$ & $3.89 e-02_{1.2 e-02}$ & $1.43 e-02_{1.8 e-03}$ & $9.04 e-03_{2.0 e-03}$ \\
\hline & 1hvl & $1.02 e+01_{5.2 e+00}$ & $9.03 e-03_{2.8 e-01}$ & $2.94 e-02_{6.0 e-03}$ & $3.93 e-027.4 e-03$ & $1.68 e-025.6 e-03$ & $1.22 e-02_{3.9 e-03}$ \\
\hline & $1 \mathrm{hvs}$ & $1.24 e+01_{5.2 e+00}$ & $3.14 e-02_{1.6 e-01}$ & $3.62 e-025.8 e-03$ & $4.87 e-028.9 e-03$ & $2.74 e-025.9 e-03$ & $1.49 e-024.0 e-03$ \\
\hline & 1hwr & $1.79 e+00_{1.0 e+01}$ & $8.70 e-037.8 e-04$ & $2.65 e-029.2 e-03$ & $3.37 e-02_{1.3 e-02}$ & $1.89 e-027.0 e-03$ & $9.61 e-03_{2.0 e-03}$ \\
\hline & lody & $7.11 e+00_{9.2 e-01}$ & $2.23 e-01_{3.3 e-01}$ & $6.13 e-027.5 e-03$ & $7.17 e-029.7 e-03$ & $4.90 e-02_{1.7 e-02}$ & $3.85 e-02_{1.4 e-02}$ \\
\hline & $1 \mathrm{vij}$ & $5.75 e+00_{3.0 e+00}$ & $5.62 e-024.3 e-02$ & $9.09 e-02_{3.3 e-02}$ & $1.10 e-01_{1.7 e-02}$ & $6.02 e-024.3 e-02$ & $5.72 e-023.9 e-02$ \\
\hline & 1 vik & $7.48 e+00_{1.0 e+00}$ & $2.77 e-02_{1.3 e-02}$ & $4.60 e-024.7 e-03$ & $5.76 e-02_{1.3 e-02}$ & $4.25 e-029.3 e-03$ & $3.12 e-02_{4.1 e-03}$ \\
\hline & 3tlh & $4.31 e-01_{7.5 e-01}$ & $8.39 e-02_{1.8 e-01}$ & $6.18 e-02_{1.1 e-02}$ & $7.18 e-02_{7.0 e-03}$ & $6.73 e-02_{1.2 e-02}$ & $5.14 e-02_{1.4 e-02}$ \\
\hline & $7 \mathrm{hvp}$ & $6.98 e-028.7 e-02$ & $1.55 e-02_{1.6 e-02}$ & $9.05 e-02_{1.6 e-02}$ & $9.87 e-02_{1.1 e-02}$ & $8.50 e-02_{1.6 e-02}$ & $7.28 e-02_{2.5 e-02}$ \\
\hline & $8 \mathrm{hvp}$ & $5.13 e-024.1 e-02$ & $2.45 e-02_{1.1 e-02}$ & $8.06 e-02_{1.6 e-02}$ & $7.77 e-02_{1.2 e-02}$ & $4.65 e-02_{1.5 e-02}$ & $4.88 e-02_{1.6 e-02}$ \\
\hline & 9 hvp & $3.37 e+00_{6.9 e+00}$ & $9.55 e-02_{1.5 e-01}$ & $7.49 e-02_{2.0 e-02}$ & $1.10 e-01_{3.1 e-02}$ & $6.40 e-02_{1.5 e-02}$ & $4.73 e-02_{6.8 e-03}$ \\
\hline \multirow{14}{*}{ Cyclic-urea } & 1 bv7 & $5.89 e+00_{1.6 e+00}$ & $2.86 e-023.5 e-02$ & $1.03 e-01_{4.7 e-02}$ & $1.29 e-01_{3.2 e-02}$ & $1.13 e-01_{5.1 e-02}$ & $5.24 e-02_{3.2 e-02}$ \\
\hline & 1bv9 & $1.03 e+01_{1.4 e+00}$ & $1.40 e-025.2 e-02$ & $1.50 e-01_{3.7 e-02}$ & $1.73 e-01_{3.9 e-02}$ & $1.27 e-01_{5.1 e-02}$ & $9.04 e-02_{6.0 e-02}$ \\
\hline & 1bwa & $6.29 e+00_{2.3 e+00}$ & $3.73 e-02_{2.7 e-02}$ & $8.84 e-022.9 e-02$ & $1.08 e-01_{1.9 e-02}$ & $6.69 e-022_{3.1 e-02}$ & $5.58 e-02_{2.6 e-02}$ \\
\hline & $1 \mathrm{bwb}$ & $7.24 e+00_{3.2 e+00}$ & $3.09 e-024.6 e-02$ & $3.69 e-02_{1.7 e-02}$ & $6.25 e-02_{2.3 e-02}$ & $2.90 e-02_{1.6 e-02}$ & $1.45 e-02_{8.0 e-03}$ \\
\hline & $1 \mathrm{dmp}$ & $1.17 e+01_{1.7 e+00}$ & $3.58 e-02_{2.7 e-02}$ & $4.79 e-02_{5.7 e-03}$ & $5.40 e-02_{3.6 e-03}$ & $4.43 e-026.7 e-03$ & $3.86 e-02_{1.3 e-02}$ \\
\hline & $\lg 35$ & $7.24 e+00_{1.5 e+00}$ & $1.89 e-01_{4.6 e-01}$ & $4.12 e-02_{1.2 e-02}$ & $5.26 e-02_{1.2 e-02}$ & $3.45 e-022_{1.1 e-02}$ & $2.43 e-02_{8.0 e-03}$ \\
\hline & 1 hpo & $2.71 e+00_{1.8 e+00}$ & $4.60 e-03_{1.4 e+00}$ & $2.36 e-02_{1.0 e-02}$ & $3.95 e-02_{1.6 e-02}$ & $1.61 e-02_{6.6 e-03}$ & $7.42 e-03_{3.8 e-03}$ \\
\hline & $1 \mathrm{mes}$ & $1.25 e+01_{3.5 e+00}$ & $4.50 e-02_{1.6 e-01}$ & $5.82 e-02_{4.9 e-02}$ & $6.84 e-02_{4.7 e-02}$ & $4.90 e-02_{3.8 e-02}$ & $1.76 e-02_{2.3 e-02}$ \\
\hline & $1 \mathrm{meu}$ & $8.64 e+00_{3.7 e+00}$ & $7.19 e-022_{1.5 e-01}$ & $5.92 e-02.7 e-02$ & $7.71 e-025.1 e-02$ & $5.07 e-023.5 e-02$ & $3.30 e-027.2 e-03$ \\
\hline & 1pro & $1.64 e+01_{3.4 e+00}$ & $1.87 e-02_{5.3 e-02}$ & $5.23 e-02_{2.3 e-02}$ & $5.61 e-02_{1.3 e-02}$ & $6.02 e-02_{2.1 e-02}$ & $3.02 e-02_{3.5 e-02}$ \\
\hline & $1 \mathrm{qbr}$ & $6.25 e+00_{1.4 e+00}$ & $4.02 e-024.1 e+00$ & $7.63 e-02_{3.5 e-02}$ & $1.16 e-01_{4.1 e-02}$ & $6.88 e-02.5 e-02$ & $5.33 e-02_{2.0 e-02}$ \\
\hline & 1qbt & $8.35 e+00_{1.6 e+00}$ & $3.33 e-02_{1.4 e-01}$ & $9.73 e-02_{2.9 e-02}$ & $1.31 e-01_{4.1 e-02}$ & $8.49 e-02_{2.3 e-02}$ & $5.40 e-02_{1.5 e-02}$ \\
\hline & 1qbu & $6.54 e+00_{2.5 e+00}$ & $3.60 e-02_{1.0 e+00}$ & $7.18 e-02_{2.0 e-02}$ & $9.83 e-022.7 e-02$ & $9.45 e-024.2 e-02$ & $5.58 e-023.5 e-02$ \\
\hline & 7upj & $8.45 e-01_{1.4 e+00}$ & $6.11 e-03_{1.7 e-02}$ & $1.84 e-02_{7.8 e-03}$ & $2.59 e-02_{1.1 e-02}$ & $1.90 e-02_{6.6 e-03}$ & $1.10 e-02_{6.6 e-03}$ \\
\hline
\end{tabular}


Table 5

Average Friedman's rankings with Holm's Adjusted p-values (0.05) of compared algorithms for the test set of 75 docking instances in terms of $I_{H V}$ and $I_{I G D+}$. Symbol * indicates the control algorithm, which in this case is SMPSOhv.

\begin{tabular}{|c|c|c|c|c|c|}
\hline \multicolumn{3}{|c|}{ Hypervolume $\left(I_{H V}\right)$} & \multicolumn{3}{|c|}{ Hypervolume $\left(I_{I G D+}\right)$} \\
\hline Algorithm & Fried. & Holm's $_{a p v}$ & Algorithm & Fried. & Holm's ${ }_{a p v}$ \\
\hline *SMPSOhv & 1.77 & - & ${ }^{*}$ SMPSOhv & 1.70 & - \\
\hline MPSO/D & 1.97 & $5.26 \mathrm{e}-01$ & MPSO/D & 2.23 & $8.08 \mathrm{e}-02$ \\
\hline SMPSOD & 3.01 & $1.08 \mathrm{e}-04$ & SMPSOD & 2.86 & $2.92 \mathrm{e}-04$ \\
\hline SMPSO & 3.79 & $1.31 \mathrm{e}-10$ & SMPSOC & 1.31 & $2.42 \mathrm{e}-10$ \\
\hline SMPSOC & 3.91 & $4.43 e-24$ & SMPSO & 4.78 & $2.66 \mathrm{e}-23$ \\
\hline OMOPSO & 5.52 & $7.08 \mathrm{e}-34$ & OMOPSO & 5.67 & $1.33 e-38$ \\
\hline
\end{tabular}

16 ones. It is worth noting that some results have a $I_{H V}$ equal to zero. This happens when all the points of the fronts produced are dominated by the reference point. In this regard, for a high number of instances, OMOPSO shows $I_{H V}$ equal to zero, whereas for practically all the SMPSO versions and MPSO/D, the hypervolume values are higher than zero, which indicates that they are all able to produce solutions within the limits of the reference point.

Nevertheless, we can still identify some instances for which OMOPSO shows the second best hypervolume: $1 \mathrm{bdl}, 1 \mathrm{hef}, 2 \mathrm{bpv}, 1 \mathrm{hos}$, 7hvp, and 8hvp, so probably these compounds are characterized with different fitness landscapes more adapted to the learning procedure of this algorithm.

Apart from this, it is intuitively obvious that SMPSOhv could show a good performance in terms of $I_{H V}$, as this algorithm uses the hypervolume indicator to guide the convergence of the population. For this reason, the $I_{I G D+}$ is also used here as an alternative metric to avoid the possible bias that hypervolume could induce in the results. $I_{I G D+}$ is a refined version of the inverted generational distance (IGD) [15], which measures the average distance from each reference point to the nearest solution in the solution set. It can be viewed as an approximate distance from the Pareto front to the solution set in the objective space, so the lower the IGD+value, the better the front approximation.

To test this, Table 4 contains the median and interquartile range of the computed distributions (out of 31 independent runs) in terms of $I_{I G D+}$, for the set of 75 docking instances and for the six algorithms compared. In general, it is clearly observable that $I_{I G D+}$ results are quite similar to those obtained in terms of $I_{H V}$, which leads us to confirm the prominent behavior of MPSO/D and SMPSOhv in comparison with the other multi-objective PSO variants. In addition, as happens for the hypervolume indicator (Table 3), SMPSOD shows in general the third best results. OMOPSO obtains successful performance for the specific instances: 1bdl, 1hef, 2bpv, and 7hvp.

In order to assess these results with statistical confidence (in this study $p$-value $=0.05$ ), we focus on the entire distribution of each of the studied metrics by using statistical tests. Specifically, we have applied Friedman's ranking and Holm's post-hoc multi-compare tests [36] to see which algorithms are statistically worse than the control one (i.e,
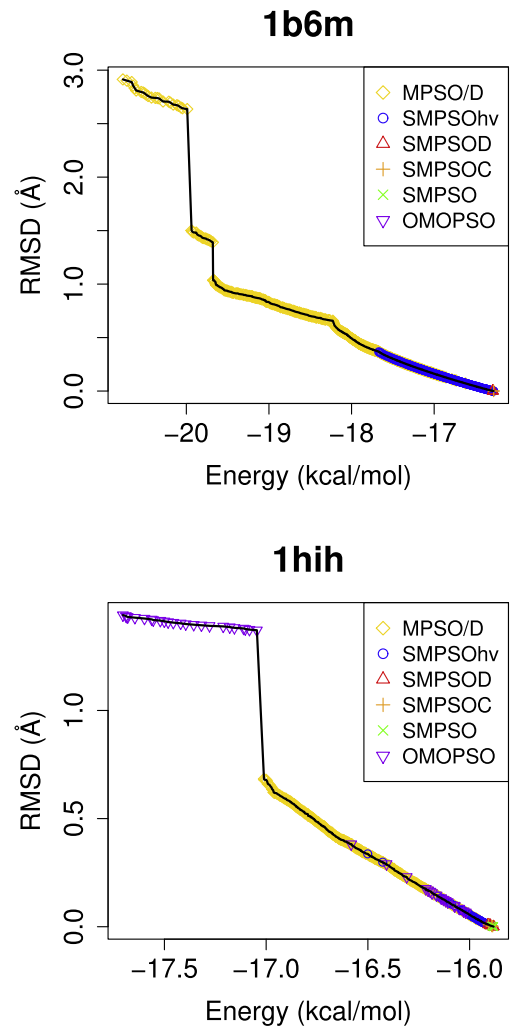

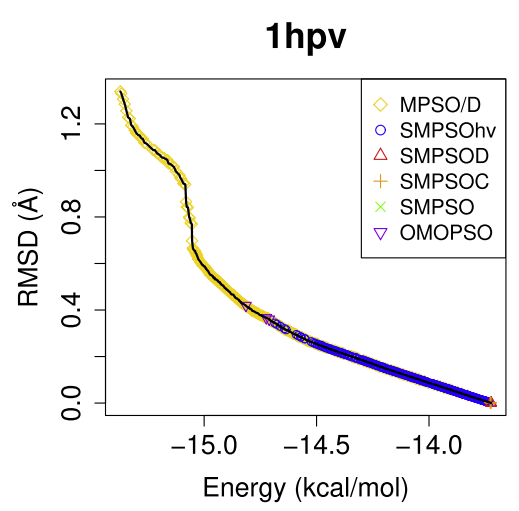

$1 \mathrm{bdr}$

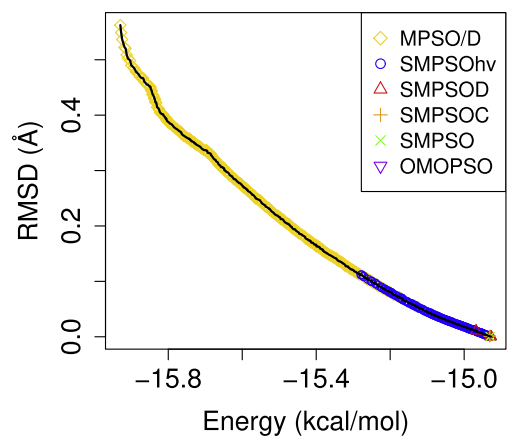

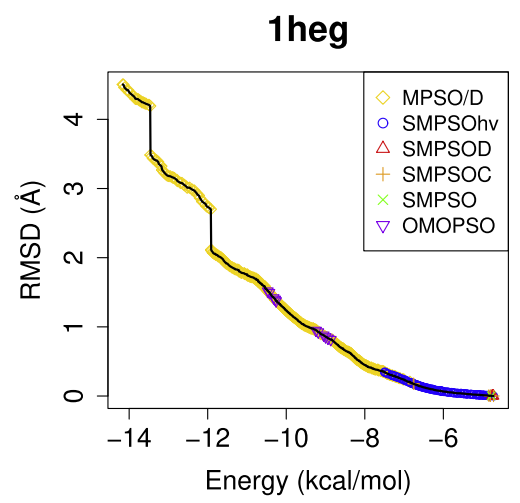

1zih

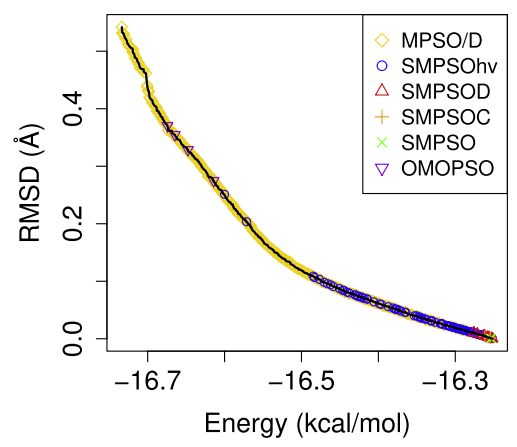

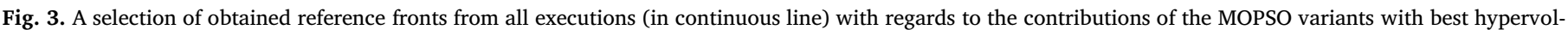
ume (in dotted lines) for small-size molecular instances: 1b6m, 1hpv, $1 \mathrm{heg}, 1 \mathrm{hih}, 1 \mathrm{bdr}$, and $1 \mathrm{zih}$. 
1hps

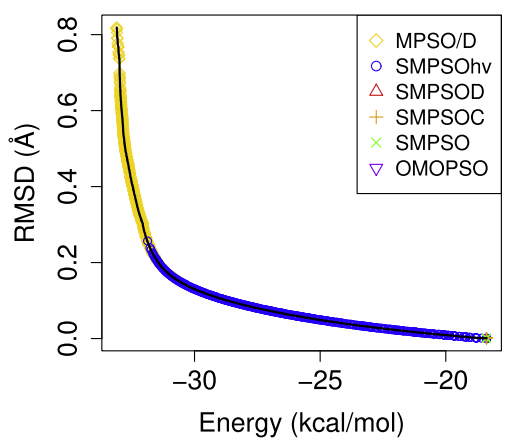

4hvp

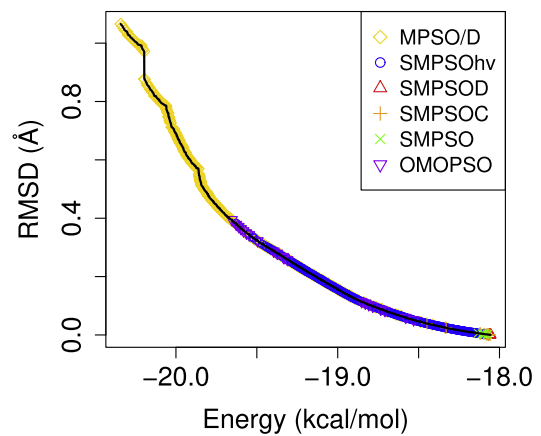

$1 b 6 p$

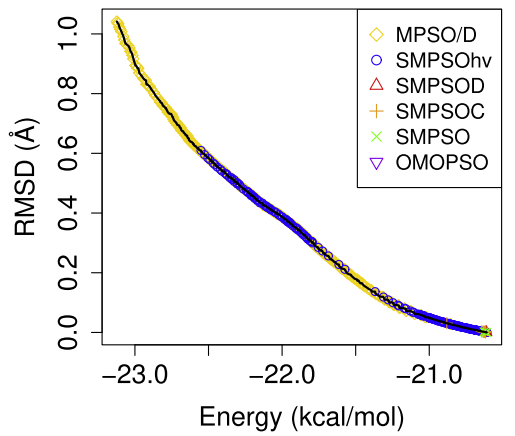

$2 b p x$

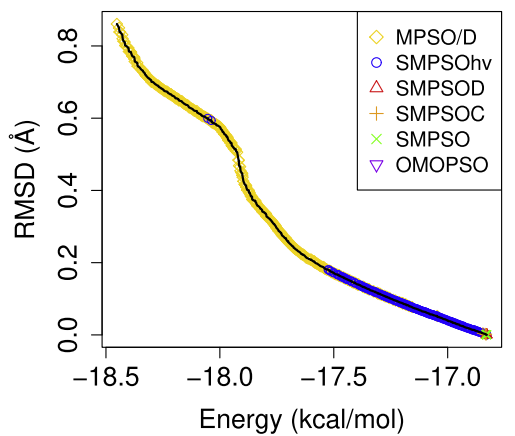

1b6k

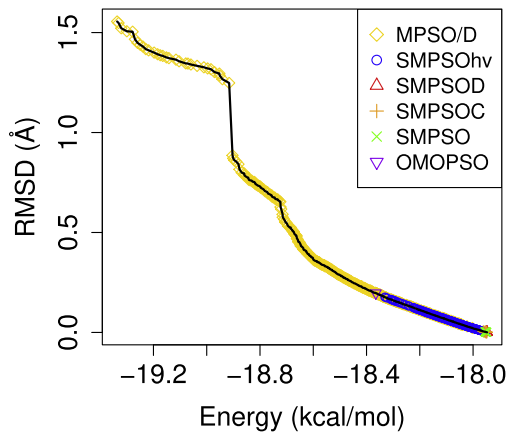

1d4I

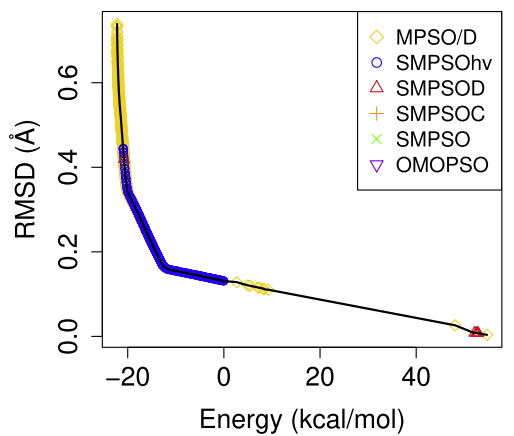

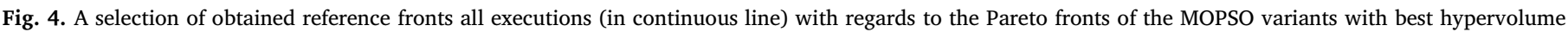
(in dotted lines) for medium-size molecular instances: 1hps, 1b6p, 1b6k, 4hvp, 2bpx, and 1d4l.

the one with best Friedman rank).

Table 5 shows that SMPSOhv is the best ranked variant according to the test of Friedman for the distributions of $I_{H V}$ and $I_{I G D+}$. Therefore, SMPSOhv is established as the control algorithm in the post-hoc Holm's tests, which is compared with the rest of algorithms. It is worth noting that, even though MPSO/D obtained the highest number of best median values (see Tables 3 and 4), the Friedman's test shows better rankings for SMPSOhv. This leads us to suggest that this variant obtains in general better distributions than MPSO/D (which behaves more biased to the median value), although without statistical confidence, as the adjusted $p$-values in accordance with Holm's test are higher than the confidence level (0.05), for the two quality indicators.

In this sense, for the remaining variants: SMPSO, SMPSOD, SMPSO, and OMOPSO; the adjusted $p$-values resulting from these comparisons are lower than the confidence level, meaning that SMPSOhv is statistically better than these algorithms. SMPSOD is ranked in third position. SMPSO and SMPSOC obtained similar rankings, although the former performed better in terms of $I_{H V}$, whereas the later obtains better ranking for $I_{I G D+}$.

To graphically illustrate this, Figs. 3-6 plot the computed reference fronts from all executions (in continuous line) with regards to the contributions to these fronts of each compared algorithm, for a selection of molecular instances with small-size, medium-size, large-size and cyclicurea, respectively. These plots have been selected as they are highly representative of the complete set molecular instances, but we also include some interesting cases to illustrate different results, such as: 1heg, 1hih, 1zih (Fig. 3), 1hwr, and 1htg (Fig. 5) and 1qbv (Fig. 6).

A general observation from these plots is that SMPSOhv and MPSO/D contribute with non-dominated solutions to the reference front in practically all the molecular complexes, although its contribution is poor for several large-size instances, like: 3 tlh and $1 \mathrm{hwr}$. These reference fronts are better covered by solutions of SMPSOD and OMOPSO, which perform optimization procedures more adapted to these special cases. In all likelihood, the replacement mechanism in SMPSOhv of non-dominated solutions with low contribution in terms of hypervolume is what makes this variant discard reference solutions prematurely, whereas SMPSOD and OMOPSO are able keep solutions in these reference fronts.

Another interesting observation in the plotted fronts lies in the number of non-dominated solutions with different values of RMSDs, which also show low energies in terms of $\mathrm{kcal} / \mathrm{mol}$. This leads us to suggest that using our multi-objective approach it is now possible to provide the decision-maker with alternative conformations of the ligands in the receptor's active sites, and with low energy values. In fact, the binding energies obtained are, in some cases, even lower than the reference molecular conformations obtained from in vivo experiments (i. e. the standard gold solutions in PDB DataBank), then giving rise to more stable complexes.

An example of this can be taken from small-size instances 1 heg and 1hih in Fig. 3, for which there are solutions with relatively high values of RMSDs $(>\approx 1.5 \AA)$, although computing low energies: -14.16 and $-17.50 \mathrm{kcal} / \mathrm{mol}$, respectively. In contrast, these fronts also contains solutions with RMSD values close to $0 \AA$ (with a precision error of 10e-4), i.e., they are quite similar to the gold standard conformations, although the former obtains more stable conformations in terms of binding energy and higher RMSD scores. This means that we are now able to discover other different ligand-receptor conformations with lower energies than those obtained from the ligand-protein crystallographic structure of the in vivo experiments.

In summary, SMPSOhv and MPSO/D show the overall best behavior followed by SMPSOD, SMPSOC, SMPSO, and OMOPSO. Intuitively, SMPSOhv obtains the best $I_{H V}$ as it performs a leader selection method of non-dominated solutions (from the external archive) with largest hypervolume contributions. That is, the particles in the swarm are guided by leaders with large hypervolume contributions, which enables SMPSOhv to obtain high values of $I_{H V}$. Moreover, such an efficient 
1vik

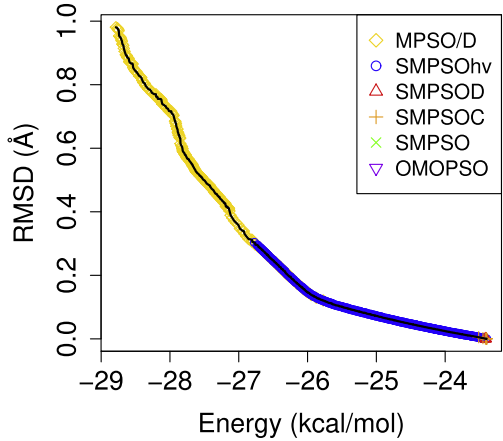

1hvi

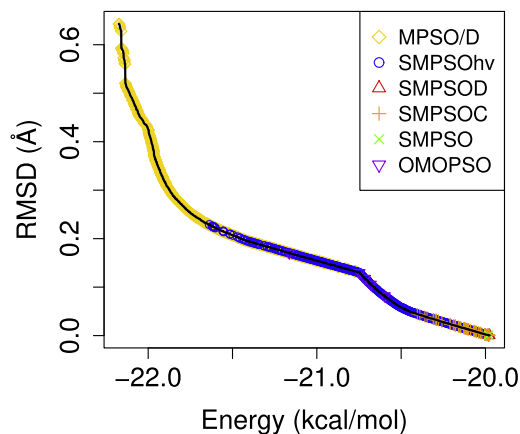

1 a94

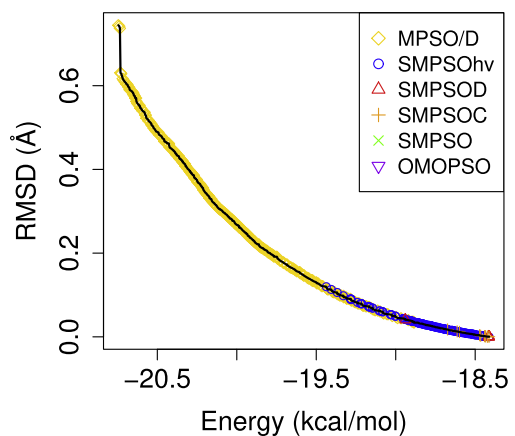

$1 \mathrm{hwr}$

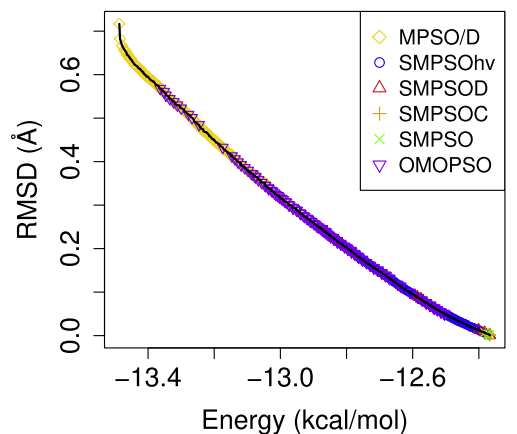

3tlh

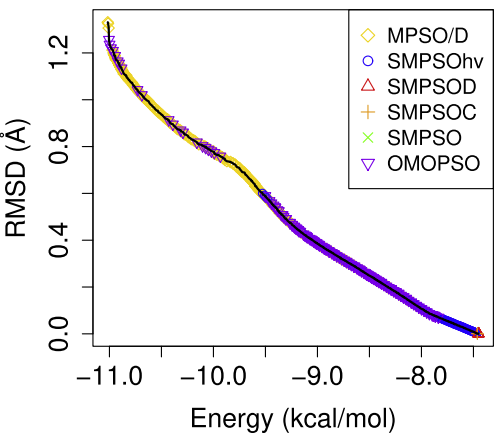

1htg

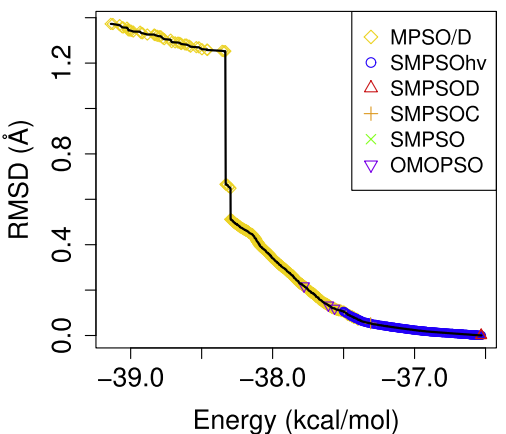

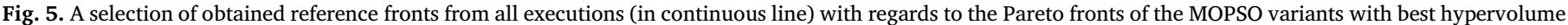
(in dotted lines) for large-size molecular instances: 1vik, 1a94, 1hos, 1hvj, 1hwr, and $1 \mathrm{htg}$.

1 hpo

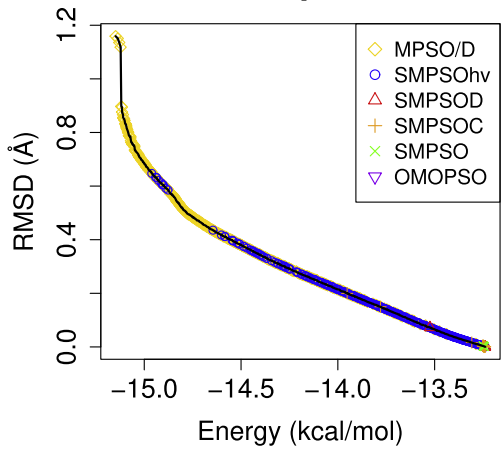

1 bwa

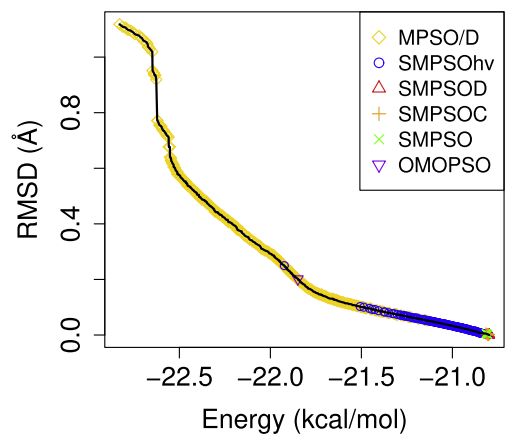

1qbt

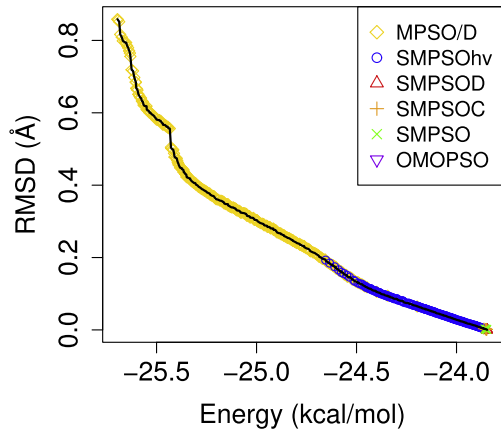

1 bwb

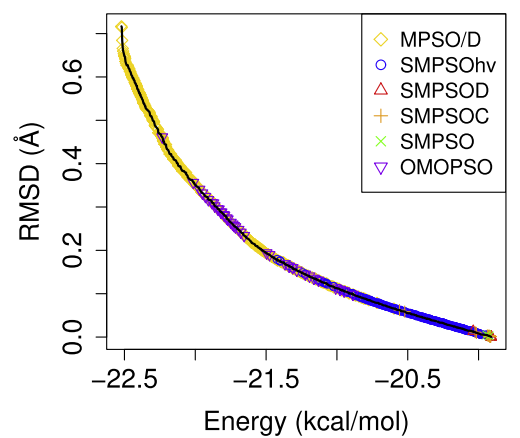

1g35

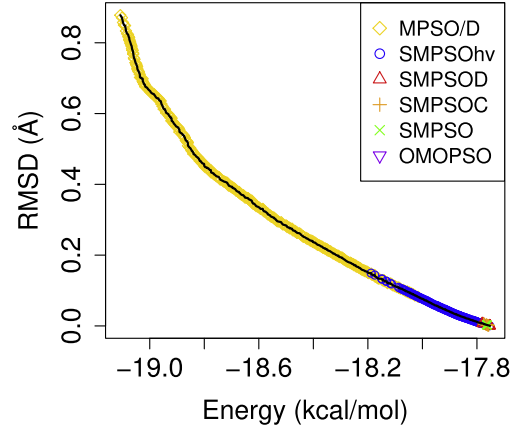

1qbu

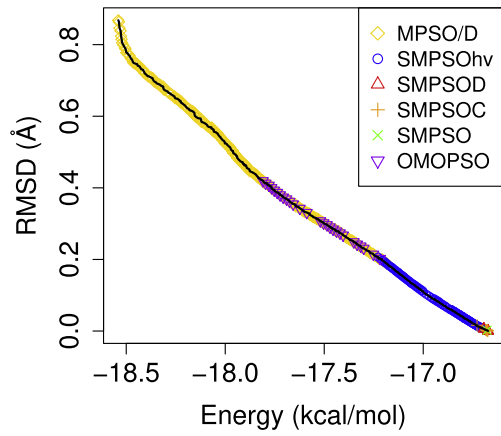

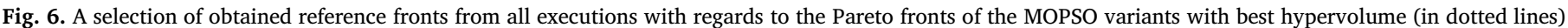
for cyclic-urea molecular instances: 1hpo, 1qbt, 1g35, 1bwb, 1qbu and 1bwa. 


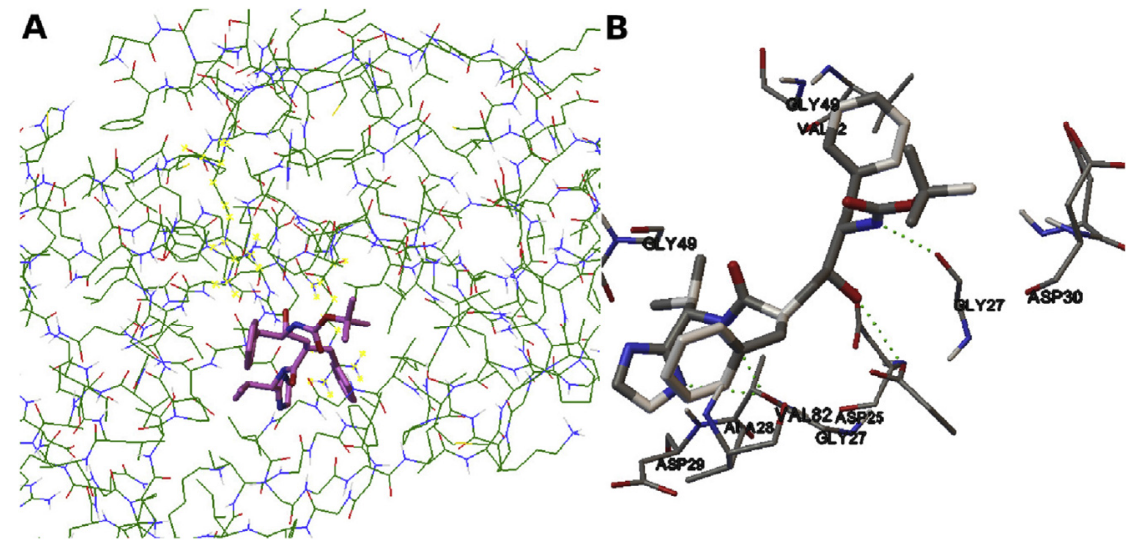

Fig. 7. Image A represents the chimeric HIV-protease displayed as sticks. The two residues of ARG-8 for HIV-protease monomer A and B are selected in yellow. ARG- 8 are two aminoacids located close to the HIV-protease active site. Image B shows the molecular interactions between ligandreceptor. The computed ligand is represented in green. The $\mathrm{H}$-bonds are represented with green spheres. The residues involved in the ligand-receptor interactions are also labeled, including the residues that are closer to this interaction. (For interpretation of the references to colour in this figure legend, the reader is referred to the Web version of this article.)

behavior is also observed for $I_{I D G+}$, so that it could be claimed that this variant is also able to obtain prominent Pareto front approximations independently to the computation of the hypervolume values. Other variants like SMPSOD and OMOPSO are also able to obtain interesting solutions from the point of view of the molecular docking modeling, allowing us to see alternative conformations with lower energies than those of the gold standard solutions.

In terms of computational cost, an average running time of $4 \mathrm{~min}$ per independent run is observed for each algorithm and molecular instance. This is an acceptable time in molecular docking procedures as they usually take place in off-line simulations to detect the best ligand's conformation to a receptor.

\subsection{Analysis of the ligand binding sites and molecular interactions}

The second analysis in this study consists in exploring the resulting molecular conformations from a biological point of view. With this aim, we have selected two instances: $1 b d r$ (small-size) and $1 g 35$ (cyclic urea), which have been widely studied in the specialized literature $[1,35,37]$.

In this regard, according to the results obtained by Morris et al. [26], the LGA algorithm (described in Section 2) fails to find the ligand's pose in the molecular docking simulations when freedom degrees are applied to the small-size HIV-protease ligands' bonds. The application of flexibility involves a larger number of ligand conformations possibilities due to the small size of the ligands. The instance $1 b d r$ belongs to this group of instances, where the size of the HIV-protease inhibitor is smaller than the other ligand sets. The application of flexibility and its influence on the search space does not only depend on the ligand size, but also on the ligand chemical structure. The instance $1 g 35$ has as ligand a cyclic urea inhibitor, which has a conformation that allows to bind correctly to the HIV-protease active site. These larger ligands are forced to be, at least, partially inside the HIV-protease active site tunnel due to their size. However, with the application of flexibility to these ligands, it can be difficult to find the perfect ligand pose to the HIV-protease receptor.
In this analysis, two different solutions have been chosen from the extremes of the fronts obtained for $1 b d r$ and $1 g 35$. These solutions are analyzed in terms of ligand binding site and molecular interactions, as follows:

- 1bdr. This molecule corresponds to the complex HIV-1 protease with inhibitor SB203386, a tripeptide analog inhibitor. This chimeric HIV-protease crystal is formed by replacing aminoacids in residues in the HIV type 1 with the corresponding residues from the HIV type 2 (31-37) and in the active site. It contains the mutations t31s, 133v, e34a, e35g, m36i, and s37e. A previous study [37] reported that these mutations can affect the dimensions of the HIV-protease active site, and hence the interaction HIV-proteaseinhibitors. Given the accumulation of mutations in this therapeutic target and the difference of response of HIV patients to the treatment with new HIV-protease inhibitors, it is necessary to conduct studies in silico with accurate molecular docking techniques to observe how these new mutations could affect to the inhibitor interactions.

In accordance with the resulting fronts plotted in Fig. 3, we have chosen the non-dominated solution that has an intermolecular energy of $-15.28 \mathrm{kcal} / \mathrm{mol}$ and an RMSD of $0.11 \AA$. Image A in Fig. 7 shows the co-crystallized and the predicted ligands' conformations. As illustrated, the two ligands overlap, which is explained by the low RMSD value. The reference and computed ligands are represented in pink and green sticks, respectively. Both structures are located in the active site of the HIV-protease, which is a shaped-tunnel. The ARG8 residues are marked in yellow. Image B in Fig. 7 shows the molecular ligand-receptor interactions in which H-bonds are represented in green. The N8 of SB203386 interacts with the oxygen atom of the GLY27 amide of the HIV-protease monomer A through an H-bond. The $\mathrm{H}$ atom of group $-\mathrm{OH}$ of ligand's atom $\mathrm{O} 18$ interacts to the $\mathrm{O}$ atom of the group $-\mathrm{OH}$ of ASP25 side chain of the HIV-protease monomer B through a H-bond. The N30 AND N39 atoms of ligand interact with the oxygen of GLY27 amide of HIVprotease monomer B. The solutions obtained show that the catalytic
A

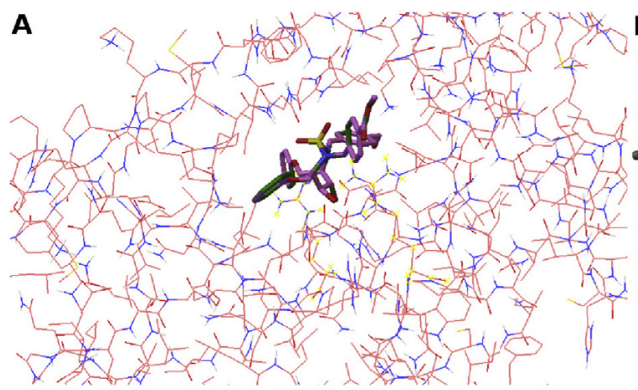

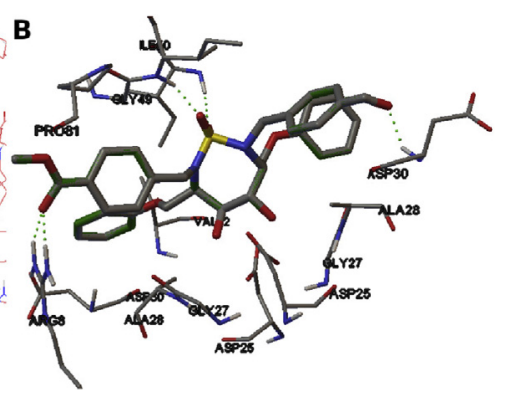

Fig. 8. Image A represents the reference and computed ligand in green and pink, respectively. The ligands and the HIV-protease are represented as sticks. The two ligands' conformations overlap. The two ARG8 side chains are located in the HIV-protease tunnel are selected in yellow. Image B shows the molecular interactions. The H-bonds are represented with green spheres. The residues involved in the ligand-receptor interactions are also labeled, including the residues that are closer to the receptor-ligand interaction. (For interpretation of the references to colour in this figure legend, the reader is referred to the Web version of this article.) 
aminoacids (ASP25 and GLY27) of the monomers A and B are involved in the molecular ligand-receptor interaction. These results are in agreement with the studies performed in Refs. [1] and [37].

- 1g35. This instance corresponds to the complex HIV-protease with inhibitor AHA024, a sulfamide compound. In Ref. [35], the authors performed a test to demonstrate that the non-symmetric sulfamide inhibitors should be more potent than their corresponding symmetric sulfamide inhibitors. This way, as our multi-objective approach enables the decision-maker to choose from a wide range of solutions, it can be useful in those cases in which the binding sites of new candidate compounds are being tested. In line with the plotted fronts in Fig. 5 for 1g35, we have chosen the non-dominated solution that has an intermolecular energy of $-17.89 \mathrm{kcal} / \mathrm{mol}$ and an RMSD value of $0.15 \AA$.

Image A of Fig. 8 shows that the co-crystallized and predicted ligands (in green and pink, respectively) are bound to the shaped-tunnel where the HIV-protease binding site is found. The two flexible ARG8 are marked in yellow. It can be observed the fact that although the solution has a higher RMSD value than the other solutions from the front, the two ligands' conformations are overlapping, what indicates the accuracy of the predicted ligand's conformation. Image B shows the molecular interactions of AHA024/HIV-protease. The predicted Hbonds are represented with green spheres. The $\mathrm{O} 80$ and $\mathrm{O} 15$ interact to the GLY49 and ILE50, respectively through two H-bonds. The O47 of the ligand interacts to ASP30 amide through an H-bond as reported in Ref. [35]. The 044 interacts to the ARG8 side-chain through H-bonds. This computed ligand's conformation is interesting from a pharmacological point of view, since the ligand interacts with ARG8, which is located at the end of the active site tunnel. Although there are solutions with even lower RMSDs (lower than $0.15 \AA$ ), this predicted ligand interaction with the ARG- 8 residue allows the compound to adopt a pose inside the active site tunnel, blocking the binding of the substrate to HIV-protease. The lower intermolecular energy leads us to verify that the complex is very stable in terms of energy.

\section{Conclusions}

This study has analyzed a number of variants of multi-objective particle swarm optimizers, based on different archiving strategies, when solving the molecular docking problem. We have extended our previous research by assessing the performance of the different versions of SMPSO and two other optimizers, OMOPSO and MPSO/D, when tackling an extensive benchmark of 75 PDB structures (instances). The problem has been formulated as a bi-objective optimization problem, by minimizing the binding energy and the RMSD difference in the coordinates of ligands.

After a thorough experimentation phase, the following main conclusions can be drawn from the analysis:

- Our study confirms that SMPSOhv and MPSO/D show the overall best performance in terms of $I_{H V}$ and $I_{I D G+}$, followed by SMPSOD, SMPSOC, SMPSO and OMOPSO. SMPSOhv performs a leader selection method of those non-dominated solutions (from the external archive) with the largest hypervolume contributions, which seems to be responsible for the best diversity and convergence values in this comparison.

- The archive-less approaches based on objective space decomposition, MPSO/D and SMPSOD, appear as prominent optimizers when dealing with molecular docking, concretely MPSO/D. Both algorithms are able to cover the reference front with non-dominated solutions that compute very low binding energy, but with relatively high values of RMSD. This encourages us to suggest new alternative conformations with lower energies than those of the gold standard solutions.
- SMPSO, SMPSOC, and OMOPSO show moderate results, although they obtain interesting solutions from the point of view of the ligand-protein interactions. For several compounds: 1bdl, 1a9m, $1 \mathrm{bdl}$, 1hef, 2bpv, 1hps, 1hos, 7hvp, and 8hvp, these algorithms performed efficiently. SMPSO and SMPSOC obtained similar rankings, although the former performed better in terms of $I_{H V}$, whereas the later obtains better ranking for $I_{I G D+}$.

- As a matter of biological validation, a specific analysis of the resulting conformations for molecules $1 b d r$ and $1 g 35$ show overlapping ligands with regards to the specialized literature, where these complexes are studied [1,35,37]. In fact, these solutions are shown to obtain energetically stable ligand-receptor complexes, as reported in Ref. [35]. This indicates that our predicted ligand interactions with the flexible residue allow the compound to adopt a pose inside the active site tunnel, hence blocking the binding of the substrate to HIV-protease. The lower intermolecular energy leads us to verity that the complexes are stable in terms of energy.

As for future work, we are currently designing new algorithmic proposals by combining new leader selection and archiving strategies, to find solutions covering the full Pareto front. In this regard, the use of unbounded archives and restarting methods could be beneficial for experiments in which other alternative active sites in compounds are required to be explored and discovered. We are also interested in evaluating the robustness of new MOPSO proposals in special cases, in which the active site of a given therapeutic target mutates and causes multidrug resistance.

\section{Acknowledgements}

This work is partially funded by Grants TIN2017-86049-R, TIN201458304 (Ministerio de Ciencia e Innovación) and P12-TIC-1519 (Plan Andaluz I+D+I). José García-Nieto is recipient of a Post-Doctoral fellowship of "Captación de Talento para la Investigación" at Universidad de Málaga.

\section{References}

[1] Sherin S. Abdel-Meguid, Brian W. Metcalf, Thomas J. Carr, Peter Demarsh, Renee L. DesJarlais, Seth Fisher, David W. Green, Lucinda Ivanoff, Dennis M. Lambert, Krishna H.M. Murthy, Stephen R. Petteway Jr., William J. Pitts, Thaddeus A. Tomaszek Jr., Evon Winborne, Baoguang Zhao, Geoffrey B. Dreyer, Thomas D. Meekllyo, An orally bioavailable hiv-1 protease inhibitor containing an imidazole-derived peptide bond replacement: crystallographic and pharmacokinetic analysis, Biochemistry 33 (39) (1994) 11671-11677.

[2] E. Atilgan, J. Hu, Efficient protein-ligand docking using sustainable evolutionary algorithm, in: GECCO, 2010, pp. 211-212.

[3] Cai Dai, Yuping Wang, Miao Ye, A new multi-objective particle swarm optimization algorithm based on decomposition, Inf. Sci. 325 (2016) 541-557.

[4] H.-M. Chen, B.-F. Liu, H.-L. Huang, S.-F. Hwang, S.-Y. Ho, Sodock: swarm optimization for highly flexible protein-ligand docking, J. Comput. Chem. 28 (2) (2007) 612-623.

[5] M. Clerc, J. Kennedy, The particle swarm - explosion, stability, and convergence in a multidimensional complex space, IEEE Trans. Evol. Comput. 6 (1) (2002) 58-73.

[6] C.A. Coello, G. Toscano, M.S. Lechuga, Handling multiple objectives with particle swarm optimization, IEEE Trans. Evol. Comput. 8 (3) (2004) 3.

[7] K. Deb, A. Pratap, S. Agarwal, T. Meyarivan, A fast and elitist multiobjective genetic algorithm: NSGA-II, IEEE Trans. Evol. Comput. 6 (2) (2002) 182-197.

[8] J.J. Durillo, A.J. Nebro, C. Coello Coello, J. Garcia-Nieto, F. Luna, E. Alba, A study of multiobjective metaheuristics when solving parameter scalable problems, IEEE Trans. Evol. Comput. 14 (4) (2010) 618-635.

[9] Juan J. Durillo, José García-Nieto, Antonio J. Nebro, Carlos A. Coello Coello, Francisco Luna, Enrique Alba, in: 5th Int. Conf. Evol. Multi-criterion Optimization, Chapter Multi-objective Particle Swarm Optimizers: an Experimental Comparison, Springer, 2009, pp. 495-509.

[10] M.J. García-Godoy, E. López-Camacho, J. García Nieto, A.J. Nebro, J.F. Aldana-Montes, Solving molecular docking problems with multi-objective metaheuristics, Molecules 20 (6) (2015) 10154-10183.

[11] José García-Nieto, Esteban López-Camacho, María Jesús García Godoy, Antonio J. Nebro, Juan J. Durillo, José F. Aldana-Montes, A study of archiving strategies in multi-objective pso for molecular docking, in: Swarm Intelligence: 10th Int. Conf., ANTS 2016, Brussels, Belgium, Sept, Springer Int. Publishing, 2016, pp. 40-52.

[12] A. Grosdidier, V. Zoete, O. Michielin, EADock: docking of small molecules into protein active sites with a multiobjective evolutionary optimization, Proteins 67 (4) (2007) 1010-1025. 
[13] Junfeng Gu, Xu Yang, Ling Kang, Jinying Wu, Xicheng Wang, MoDock: a multi-objective strategy improves the accuracy for molecular docking, Algs. Mol. Biol. 10 (2015) 8.

[14] Ruth Huey, Garrett M. Morris, Arthur J. Olson, David S. Goodsell, A semiempirical free energy force field with charge-based desolvation, J. Comput. Chem. 28 (6) (2007) 1145-1152.

[15] H. Ishibuchi, H. Masuda, Nojima Y, Sensitivity of performance evaluation results by inverted generational distance to reference points, in: 2016 IEEE Congress on Evolutionary Computation (CEC), 2016, pp. 1107-1114.

[16] Stefan Janson, Daniel Merkle, Martin Middendorf, Molecular docking with multi-objective particle swarm optimization, Appl. Soft Comput. 8 (1) (2008) 666-675.

[17] jMetalCpp Framework, Jmetalcpp Framework, 2012, http://sourceforge.net/ projects/jmetalcpp.

[18] J. Kennedy, R. Eberhart, Particle swarm optimization, in: IEEE International Joint Conference on Neural Networks (IJCNN), vol. 4, 1995, pp. 1942-1948. vol. 4.

[19] Y. Liu, L. Zhao, W. Li, D. Zhao, M. Song, Y. Yang, Fipsdock. A new molecular docking technique driven by fully informed swarm optimization algorithm, J. Comput. Chem. 34 (2012) 67-75.

[20] P.F. Leonhart, E. Spieler, R. Ligabue-Braun, M. Dorn, Marcio A biased random key genetic algorithm for the protein-ligand docking problem, Soft Comput. (2018) 1-22, First online.

[21] Oleg Trott, J. Olson Arthur, AutoDock Vina: improving the speed and accuracy of docking with a new scoring function, efficient optimization, and multithreading, J. Comput. Chem. 31 (2) (2010) 455-461.

[22] E. López-Camacho, M.J. García-Godoy, A.J. Nebro, J.F. Aldana-Montes, jMetalCpp: optimizing molecular docking problems with a $\mathrm{C}++$ metaheuristic framework, Bioinformatics 30 (3) (February 2014) 437-438.

[23] Esteban López-Camacho, María Jesús García-Godoy, José García-Nieto, Antonio J. Nebro, José F. Aldana-Montes, Solving molecular flexible docking problems with metaheuristics: a comparative study, Appl. Soft Comput. 28 (2015) 379-393.

[24] Esteban López-Camacho, María Jesús García-Godoy, José García-Nieto, Antonio J. Nebro, José F. Aldana-Montes, A new multi-objective approach for molecular docking based on rmsd and binding energy, in: In LNCB, 3rd Int. Conf. On Algorithms for Comp.L Biology, vol. 9702, 2016, pp. 65-77.

[25] Garrett M. Morris, David S. Goodsell, Robert S. Halliday, Ruth Huey, William E. Hart, Richard K. Belew, Arthur J. Olson, Automated docking using a lamarckian genetic algorithm and an empirical binding free energy function, J. Comput. Chem. 19 (1998) 1639-1662.

[26] Garrett M. Morris, Ruth Huey, William Lindstrom, Michel F. Sanner, Richard K. Belew, David S. Goodsell, Arthur J. Olson, AutoDock4 and AutoDockTools4: automated docking with selective receptor flexibility, J. Comput. Chem. 30 (16) (2009) 2785-2791.
[27] V. Namasivayam, R. Günther, Research article: pso@autodock: a fast flexible molecular docking program based on swarm intelligence, Chem. Biol. Drug Des. 70 (6) (2007) 475-484.

[28] A. Nebro, J. Durillo, J. Garcia-Nieto, C.A. Coello Coello, F. Luna, E. Alba, SMPSO: a new PSO-based metaheuristic for multi-objective optimization, in: IEEE Symp. On Comp. Intel. in Multi-criteria Decision-making, 2009, pp. 66-73.

[29] A.J. Nebro, J.J. Durillo, C.A. Coello Coello, Analysis of leader selection strategies in a MOPSO, in: IEEE Cong. On Evol. Comp. (CEC), June 2013, pp. 3153-3160.

[30] A.J. Nebro, Juan J. Durillo, M. Vergne, Redesigning the jmetal multi-objective optimization framework, in: Conf. On Genetic and Evol. Comp., GECCO Companion '15, ACM, NY, USA, 2015, pp. 1093-1100.

[31] Andrew P. Norgan, Paul K. Coffman, Jean-Pierre A. Kocher, David J. Katzmann, Carlos P. Sosa, Multilevel parallelization of AutoDock 4.2, J. Cheminf. 3 (1) (2011) 12.

[32] A. Oduguwa, A. Tiwari, S. Fiorentino, R. Roy, Multi-objective optimisation of the protein-ligand docking problem in drug discovery, in: Proceedings of the 8th Int. Conf. On Genetic and Evol. Comp, 2006, pp. 1793-1800.

[33] M. Reyes, C.A. Coello Coello, Improving PSO-based multi-objective optimization using crowding, mutation and $\epsilon$-dominance, in: 3th Int. Conf. On Evol. MultiCriterion Opt., (EMO 2005), Volume 3410 of LNCS, Springer, 2005, pp. 509-519.

[34] Angelica Sandoval-Perez, David Becerra, Diana Vanegas, Daniel Restrepo-Montoya, Fernando Niño, A multi-objective optimization energy approach to predict the ligand conformation in a docking process, in: EuroGP, 2013, pp. 181-192.

[35] W. Schaal, A. Karlsson, G. Ahlsen, J. Lindberg, H.O. Andersson, U.H. Danielson, B. Classon, T. Unge, B. Samuelsson, J. Hulten, A. Hallberg, A. Karlen, Synthesis and comparative molecular field analysis (comfa) of symmetric and nonsymmetric cyclic sulfamide hiv-1 protease inhibitors, J. Med. Chem. 44 (2) (2001) 155-169.

[36] D.J. Sheskin, Handbook of Parametric and Nonparametric Statistical Procedures, Chapman \& Hall/CRC, 2007.

[37] C. Debouck, S.S. Abdel-Meguid, M.A. Swairjo, E.M. Towler, Structural role of the 30's loop in determining the ligand specificity of the human immunodeficiency virus protease, Biochemistry 4 (1998) 10928-10936.

[38] Q. Zhang, H. Li, MOEA/D: a multiobjective evolutionary algorithm based on decomposition, IEEE Trans. Evol. Comput. 11 (6) (2007) 712-731.

[39] E. Zitzler, L. Thiele, Multiobjective evolutionary algorithms: a comparative case study and the strength pareto approach, IEEE Trans. Evol. Comput. 3 (4) (1999) 257-271. 